\title{
A spectral sequence for fusion systems
}

\author{
ANTONio DíAz RAmos
}

\begin{abstract}
We build a spectral sequence converging to the cohomology of a fusion system with a strongly closed subgroup. This spectral sequence is related to the LyndonHochschild-Serre spectral sequence and coincides with it for the case of an extension of groups. Nevertheless, the new spectral sequence applies to more general situations like finite simple groups with a strongly closed subgroup and exotic fusion systems with a strongly closed subgroup. We prove an analogue of a result of Stallings in the context of fusion preserving homomorphisms and deduce Tate's $p$-nilpotency criterion as a corollary.
\end{abstract}

55T10; 55R35, 20D20

\section{Introduction}

Let $K \unlhd G$ be a normal subgroup of the finite group $G$ and consider the extension

$$
K \rightarrow G \rightarrow G / K
$$

The Lyndon-Hochschild-Serre spectral sequence of this short exact sequence is an important tool to analyze the cohomology of $G$ with coefficients in the $\mathbb{Z} G$-module $M$. It has second page $E_{2}^{n, m}=H^{n}\left(G / K ; H^{m}(K ; M)\right)$ with $G / K$ acting on $H^{m}(K ; M)$ and converges to $H^{n+m}(G ; M)$.

Our aim in this work is to construct a related spectral sequence in the context of fusion systems. This concept was originally introduced by Puig and developed by Broto, Levi and Oliver in [3], to which we refer the reader for notation. It consists of a category $\mathcal{F}$ with objects the subgroups of a finite $p$-group $S$ and morphisms bounded by axioms that mimic properties of conjugation morphisms.

In the setup of fusion systems the concept of a short exact sequence is an evasive one: Let $\mathcal{F}$ be a fusion system over the $p$-group $S$. For a strongly $\mathcal{F}$-closed subgroup $T$ of $S$ there is a quotient fusion system $\mathcal{F} / T$; Craven [7, 5.10]. Nevertheless, in general there is no normal fusion subsystem of $\mathcal{F}$ that would play the role of the kernel of the morphism of fusion systems $\mathcal{F} \rightarrow \mathcal{F} / T$; Aschbacher [1, $8.11 \mathrm{ff}]$. So the answer to Solomon and Stancu [16, Conjecture 11] is negative and one cannot expect to construct 
a Lyndon-Hochschild-Serre spectral sequence for fusion systems. Here we are able to construct a spectral sequence that converges to the cohomology of $\mathcal{F}, H^{*}(\mathcal{F} ; M)$, where $M$ is a $\mathbb{Z}_{(p)}$-module with trivial action of $S$. Recall that $H^{*}(\mathcal{F} ; M)$ is defined [3, Section 5] as the following subring of $\mathcal{F}$-stable elements in $H^{*}(S ; M)$ :

$$
H^{*}(S ; M)^{\mathcal{F}}=\left\{z \in H^{*}(S ; M) \mid \operatorname{res}(z)=\varphi^{*}(z) \text { for each } \varphi \in \operatorname{Hom}_{\mathcal{F}}(P, S)\right\},
$$

where res: $H^{*}(S ; M) \rightarrow H^{*}(P ; M)$ is restriction in cohomology.

Theorem 1.1 Let $\mathcal{F}$ be a fusion system over the $p$-group $S, T$ a strongly $\mathcal{F}$-closed subgroup of $S$ and $M$ a $\mathbb{Z}_{(p)}$-module with trivial $S$-action. Then there is a first quadrant cohomological spectral sequence with second page

$$
E_{2}^{n, m}=H^{n}\left(S / T ; H^{m}(T ; M)\right)^{\mathcal{F}}
$$

and converging to $H^{n+m}(\mathcal{F} ; M)$.

The notation ${ }^{\mathcal{F}}$ for the second page will be fully described in Section 2, and must be thought as taking $\mathcal{F}$-stable elements in a similar way as explained for $H^{*}(\mathcal{F} ; M)$ above. Consider for each subgroup $P$ of $S$ the Lyndon-Hochschild-Serre spectral sequence of the extension

$$
P \cap T \rightarrow P \rightarrow P / P \cap T \cong P T / T
$$

converging to $H^{*}(P ; M)$. A morphism $\varphi \in \operatorname{Hom}_{\mathcal{F}}(P, Q)$ induces a morphism $\varphi^{*}$ between the spectral sequences corresponding to $Q$ and $P$. Hence we have a contravariant functor from $\mathcal{F}$ to the category of spectral sequences. Recall that a morphism in this category from $E^{\prime}$ to $E^{\prime \prime}$ is a sequence of homomorphisms of differential bigraded $\mathbb{Z}_{(p)}$-modules, $f_{k}: E_{k}^{\prime} \rightarrow E_{k}^{\prime \prime}, k \geq 0$, such that $H\left(f_{k}\right) \cong f_{k+1}$. The inverse limit spectral sequence or spectral sequence of $\mathcal{F}$-stable elements has $E_{2}^{n, m}$ entry equal to $H^{n}\left(S / T ; H^{m}(T ; M)\right)^{\mathcal{F}}$, ie, the elements $z$ from

$$
H^{n}\left(S / T ; H^{m}(T ; M)\right)
$$

such that $\varphi^{*}(z)=\operatorname{res}(z)$, where $\varphi \in \operatorname{Hom}_{\mathcal{F}}(P, S)$ and res $=\iota^{*}$ is restriction in cohomology for the inclusion $P \stackrel{\iota}{\leq} S$. Hence $H^{*}\left(S / T ; H^{*}(T ; M)\right)^{\mathcal{F}}$ is a differential graded subalgebra of the differential graded algebra $H^{*}\left(S / T ; H^{*}(T ; M)\right)$ and its differential is just restriction of the differential of the latter. This should be useful in computations. The theorem states that the abutment of this spectral sequence is $H^{*}(\mathcal{F} ; M)$.

For the case of a normal subgroup $K \unlhd G$ and $\mathcal{F}=\mathcal{F}_{S}(G)$ with $S \in \operatorname{Syl}_{p}(G)$ we have two spectral sequences converging to $H^{*}(G ; M)$. Here, $M$ is a $\mathbb{Z}_{(p)}$-module 
with trivial $G$-action (and hence trivial $S$-action). On the one hand, we have the Lyndon-Hochschild-Serre spectral sequence associated to $K \rightarrow G \rightarrow G / K$. On the other hand, we have the spectral sequence associated to $\mathcal{F}$ and the strongly $\mathcal{F}$-closed subgroup $T=K \cap S \in \operatorname{Syl}_{p}(K)$. In Section 5 we prove that the two spectral sequences are isomorphic. Note that, in particular, this shows that the Lyndon-Hochschild-Serre spectral sequence of the extension $K \rightarrow G \rightarrow G / K$ depends only on the intersection of $K$ with a Sylow $p$-subgroup of $G$.

As an application of the spectral sequence in Theorem 1.1 we prove an analogue of a result of Stallings. Meanwhile the original theorem deals with a group homomorphism, here we replace that notion by that of a fusion preserving homomorphism. This is a group homomorphism $S_{1} \rightarrow S_{2}$ between the Sylow $p$-subgroups of two fusion systems $\mathcal{F}_{1}$ and $\mathcal{F}_{2}$ such that morphisms of $\mathcal{F}_{1}$ are transformed into morphisms of $\mathcal{F}_{2}$ (see Section 6).

Theorem 1.2 (Stallings [17, page 170]) Let $\mathcal{F}_{i}$ be a fusion system over the $p$-group $S_{i}$ for $i=1,2$ and let $\phi: S_{1} \rightarrow S_{2}$ be a fusion preserving homomorphism. If the induced map in cohomology $H^{i}\left(\mathcal{F}_{2} ; \mathbb{F}_{p}\right) \rightarrow H^{i}\left(\mathcal{F}_{1} ; \mathbb{F}_{p}\right)$ is an isomorphism for $i=1$ and a monomorphism for $i=2$ then $S_{1} / \mathcal{O}_{\mathcal{F}_{1}}^{p}\left(S_{1}\right) \cong S_{2} / \mathcal{O}_{\mathcal{F}_{2}}^{p}\left(S_{2}\right)$.

The hyperfocal subgroup of $\mathcal{F}_{i}, O_{\mathcal{F}_{i}}^{p}\left(S_{i}\right),(i=1,2)$ is defined as follows:

$$
O_{\mathcal{F}_{i}}^{p}\left(S_{i}\right)=\left\langle\left[P, O^{p}\left(\operatorname{Aut}_{\mathcal{F}_{i}}(P)\right)\right] \mid P \leq S_{i}\right\rangle
$$

It is the smallest subgroup of $S_{i}$ such that the quotient of $\mathcal{F}_{i}$ over that subgroup is a p-group; see Broto, Castellana, Grodal, Levi and Oliver [2]. Hence, the conclusion of the theorem is that the largest $p$-group quotients of $\mathcal{F}_{1}$ and $\mathcal{F}_{2}$ are isomorphic. For instance, when $\mathcal{F}_{1}$ and $\mathcal{F}_{2}$ are already $p$-groups, ie, $\mathcal{F}_{i}=\mathcal{F}_{S_{i}}\left(S_{i}\right), i=1,2$, the conclusion is that $S_{1}$ and $S_{2}$ are isomorphic. This particular case is a variant of Stallings' result by Evens [10, 7.2.4]. We can also deduce fusion system versions of another result of Evens and Tate's $p$-nilpotency criterion:

Corollary 1.3 (Evens $[10,7.2 .5]$ ) Let $\mathcal{F}$ be a fusion system over the $p$-group $S$. If the map $H^{2}\left(\mathcal{F} / E_{\mathcal{F}}^{p}(S) ; \mathbb{F}_{p}\right) \rightarrow H^{2}\left(\mathcal{F} ; \mathbb{F}_{p}\right)$ is a monomorphism then $S / O_{\mathcal{F}}^{p}(S)$ is elementary abelian.

Here, the elementary focal subgroup of $\mathcal{F}$ is defined as $E_{\mathcal{F}}^{p}(S)=\Phi(S) O_{\mathcal{F}}^{p}(S)$ (Díaz, Glesser, Park and Stancu [8]), where $\Phi(S)$ is the Frattini subgroup of $S$. The conclusion of this corollary is that the largest $p$-group quotient of $\mathcal{F}$ is elementary abelian. 
Corollary 1.4 (Tate [18, Corollary on page 109]) Let $\mathcal{F}$ be a fusion system over the $p$-group $S$. If the restriction map $H^{1}\left(\mathcal{F} ; \mathbb{F}_{p}\right) \rightarrow H^{1}\left(S ; \mathbb{F}_{p}\right)$ is an isomorphism then $\mathcal{F}=\mathcal{F}_{S}(S)$.

This last result was already proven in [8] using transfer for fusion systems and in Cantarero, Scherer and Viruel [5] by topological methods. Here the proof mimics Tate's original cohomological proof that relies on the five-term exact sequence associated to the Lyndon-Hochschild-Serre spectral sequence but uses instead the spectral sequence of Theorem 1.1.

There are situations where the Lyndon-Hochschild-Serre spectral sequence is not applicable while the spectral sequence from Theorem 1.1 can be used. For instance, a classical drawback of the Lyndon-Hochschild-Serre spectral sequence is that it cannot be applied to finite simple groups. Nevertheless there are finite simple groups that do have a strongly closed $p$-subgroup: Flores and Foote [11] classified all finite groups with a strongly closed $p$-subgroup, in particular such finite simple groups. Notice that even if $\mathcal{F}$ is induced from a nonsimple finite group $\mathcal{F}=\mathcal{F}_{S}(G)$ not every strongly closed $\mathcal{F}$-subgroup $T$ of $S$ is of the form $T=K \cap S$ for some normal subgroup $K \unlhd G$ [1, Example 6.4]. This describes another circumstance where Lyndon-Hochschild-Serre does not apply but Theorem 1.1 does. As final example of this situation consider an exotic fusion system with a strongly closed $p$-subgroup. A family of such exotic fusion systems is described in Díaz, Ruiz and Viruel [9], where the authors classified all the fusion systems over $p$-groups of $p$-rank 2 ( $p$ odd).

This opens a new range of cohomology computations that can be carried out, some of which the author intends to perform in a subsequent paper. The main limitation here is that the spectral sequence from Theorem 1.1 requires knowledge of the LyndonHochschild-Serre spectral sequence of the extension of $p$-groups $T \rightarrow S \rightarrow S / T$, and these computations do not abound.

Remark 1.5 Theorem 1.1 holds for the wider class of $\mathcal{F}$-stable $\mathbb{Z}_{(p)} S$-modules, ie, for $\mathbb{Z}_{(p)} S$-modules $M$ such that for any morphism $\varphi: P \rightarrow S$ in $\mathcal{F}$ and any $p \in P$ we have $\varphi(p) \cdot m=p \cdot m$. Also, the Lyndon-Hochschild-Serre spectral sequence of $K \unlhd G$ and the spectral sequence from Theorem 1.1 for $\mathcal{F}=\mathcal{F}_{S}(G)$ and $T=S \cap K$ coincide for $G$-stable $\mathbb{Z}_{(p)} G$-modules, ie, for $\mathbb{Z}_{(p)} G$-modules $M$ such that $g^{-1} h g \cdot m=h \cdot m$ for any $h, g \in G$.

\section{Organization of the paper}

In Section 2, $\mathcal{F}$-stable elements and Mackey functors are defined and some related results introduced. In Section 3, we describe a particular cohomological Mackey functor 
that will play a central role in the construction of the spectral sequence. In Section 4, the spectral sequence is built and Theorem 1.1 is proven as Theorem 4.1. In Section 5 we compare the spectral sequence from Theorem 1.1 to the Lyndon-Hochschild-Serre spectral sequence and we give an example. In Section 6 we prove Stallings' result and some of its corollaries.

Acknowledgements I would like to thank A Viruel for several fruitful conversations when developing this paper. Also, I am grateful to P Symonds for showing me how to prove that the two spectral sequences coincide in the normal subgroup case (Theorem 5.1).

This work was supported by MCI grant RYC-2010-05663 and partially supported by FEDER-MCI grant MTM2010-18089 and Junta de Andalucía grant FQM-213

\section{Cohomology and $\mathcal{F}$-stable elements}

Throughout this section $\mathcal{F}$ denotes a fusion system over the $p$-group $S$. We start by introducing some notation: If $A: \mathcal{F} \rightarrow \mathcal{C}$ is a contravariant functor and $\mathcal{C}$ is any category then we denote the value $A(\varphi)$ by $\varphi^{*}$, where $\varphi$ is a morphism in $\mathcal{F}$. For $\varphi=\iota_{P}^{S}$, the inclusion of $P$ into $S$, we write res $:=\iota_{P}{ }^{*}$. If $\mathcal{C}$ is a complete category then we denote by $A^{\mathcal{F}}$ the inverse limit over $\mathcal{F}$ of the functor:

$$
A^{\mathcal{F}}:=\underbrace{\lim }_{\overleftarrow{\mathcal{F}}} A
$$

For the complete category $\mathrm{CCh}(\mathbf{A b})$ of (unbounded) cochain complexes we have the following favourable description of inverse limits:

Lemma 2.1 Let $A: \mathcal{F} \rightarrow \mathrm{CCh}(\mathbf{A b})$ be a contravariant functor. Then:

$$
A^{\mathcal{F}}=A(S)^{\mathcal{F}}:=\left\{z \in A(S) \mid \operatorname{res}(z)=\varphi^{*}(z) \text { for each } \varphi \in \operatorname{Hom}_{\mathcal{F}}(P, S)\right\} \subseteq A(S) .
$$

We call the elements in $A(S)^{\mathcal{F}}$ the $\mathcal{F}$-stable elements in $A(S)$. For such a functor we can consider the cohomology $H^{*}\left(A^{\mathcal{F}}\right)=H^{*}\left(A(S)^{\mathcal{F}}\right)$ of $A(S)^{\mathcal{F}} \in \mathrm{CCh}(\mathbf{A b})$. Notice that we also have functors $H^{n}(A): \mathcal{F} \rightarrow \mathbf{A b}$ obtained by taking cohomology in degree $n$. Hence we may also consider the inverse limits $H^{*}(A)^{\mathcal{F}}=H^{*}(A(S))^{\mathcal{F}}$. We are interested in functors $A$ for which taking $\mathcal{F}$-stable elements and cohomology commute. We prove in this section (Proposition 2.8) that being a cohomological Mackey functor (Definition 2.2) with values in $\mathbb{Z}_{(p)}$-modules is sufficient for this. 
Definition 2.2 Let $\mathcal{F}$ be a saturated fusion system over the $p$-group $S$ and let $\mathcal{A}$ be an abelian category. A cohomological Mackey functor for $\mathcal{F}$ over $\mathcal{A}$ is a pair of functors $(A, B): \mathcal{F} \rightarrow \mathcal{A}$ with $A: \mathcal{F} \rightarrow \mathcal{A}$ contravariant and $B: \mathcal{F} \rightarrow \mathcal{A}$ covariant such that:

(1) $A(P)=B(P)$ and $A(\varphi)=B\left(\varphi^{-1}\right)$ for each $P \leq S$ and $\varphi \in \operatorname{Hom}_{\mathcal{F}}(P, \varphi(P))$.

(2) (Identity) $A\left(c_{p}\right), B\left(c_{p}\right): A(P) \rightarrow A(P)$ are the identity morphisms for every $p \in P \leq S$, where $c_{p}: P \rightarrow P, x \mapsto p x p^{-1}$ is conjugation by $p$.

(3) (Double coset formula)

$$
A\left(\iota_{Q}^{P}\right) \circ B\left(\iota_{R}^{P}\right)=\sum_{x \in Q \backslash P / R} B\left(\iota_{Q \cap x_{R}}^{Q}\right) \circ A\left(\iota_{Q \cap{ }^{x} R}^{x_{R}}\right) \circ A\left(c_{x^{-1} \mid{ }^{x} R}\right)
$$

for $Q, R \leq P \leq S$, where $Q \backslash P / R$ are the double cosets.

(4) (Cohomological) $B\left(\iota_{P}^{Q}\right) \circ A\left(\iota_{P}^{Q}\right): A(Q) \rightarrow A(Q)$ is multiplication by $|Q: P|$ for every $P \leq Q \leq S$.

See Webb [19] for the classical definition of Mackey functors and of cohomological Mackey functors for finite groups.

Remark 2.3 In Definition 2.2 we have omitted the familiar conditions:

- (Transitivity)

$$
B\left(\iota_{Q}^{R}\right) \circ B\left(\iota_{P}^{Q}\right)=B\left(\iota_{P}^{R}\right) \quad \text { and } \quad A\left(\iota_{P}^{Q}\right) \circ A\left(\iota_{Q}^{R}\right)=A\left(\iota_{P}^{R}\right)
$$

for $P \leq Q \leq R \leq S$.

- (Conjugation)

$$
\begin{aligned}
& B\left(\iota_{P}^{Q}\right) \circ A\left(\varphi_{\mid P}\right)=A(\varphi) \circ B\left(\iota_{\varphi(P)}^{\varphi(Q)}\right), \\
& B\left(\varphi_{\mid P}\right) \circ A\left(\iota_{P}^{Q}\right)=A\left(\iota_{\varphi(P)}^{\varphi(Q)}\right) \circ B(\varphi),
\end{aligned}
$$

for $P \leq Q \leq S, \varphi \in \operatorname{Hom}_{\mathcal{F}}(Q, \varphi(Q))$.

In fact, they are consequences of the functoriality of $A$ and $B$ and of Condition (1).

We will use several times in the paper that cohomology of finite groups is a cohomological Mackey functor. For a proof of this fact see, eg, Brown [4]. 
Remark 2.4 If the maps $B\left(\iota_{P}^{Q}\right)$ for the inclusions $\iota_{P}^{Q}: P \rightarrow Q$ with $P, Q \leq S$ are given (these maps are called transfer) we can define $B$ as follows: For any morphism $\varphi \in \underset{\widetilde{\sim}}{\operatorname{Hom}_{\mathcal{F}}}(P, Q)$ define $B(\varphi): A(P) \rightarrow A(Q)$ by $B(\varphi)=B\left(\iota_{\varphi(P)}^{Q}\right) \circ A\left(\widetilde{\varphi}^{-1}\right)$ with $\widetilde{\varphi}: P \stackrel{\cong}{\rightarrow} \varphi(P)$. Such a $B$ becomes functorial if for any $P \leq Q \leq R$ we have $B\left(\iota_{Q}^{R}\right) \circ B\left(\iota_{P}^{Q}\right)=B\left(\iota_{P}^{R}\right)$ and for any $P \leq Q \stackrel{\varphi}{\rightarrow} \varphi(Q)$ we have

$$
B\left(\iota_{P}^{Q}\right) \circ A\left(\varphi_{\mid P}\right)=A(\varphi) \circ B\left(\iota_{\varphi(P)}^{\varphi(Q)}\right) .
$$

Before proving the main result of this section we need to introduce $(G, H)$-bisets: Sets with commuting free right $G$-action and free left $H$-action. Every $(G, H)$-biset $\Omega$ can be decomposed into a disjoint union of transitive $(G, H)$-bisets of the form

$$
H \times{ }_{\varphi} G=H \times G / \sim,
$$

with $K \leq G, \varphi: K \rightarrow H$ a monomorphism and

$$
(h, k g) \sim(h \varphi(k), g)
$$

for $h \in H, g \in G$ and $k \in K$. A saturated fusion system gives rise to a special type of biset:

Proposition 2.5 [3, Proposition 5.5] For any saturated fusion system $\mathcal{F}$ over a $p-$ group $S$, there is an $(S, S)$-biset $\Omega$ with the following properties:

(a) Each transitive component of $\Omega$ is of the form $S \times{ }_{\varphi} S$ for some $P \leq S$ and $\varphi \in \operatorname{Hom}_{\mathcal{F}}(P, S)$.

(b) For each $P \leq S$ and each $\varphi \in \operatorname{Hom}_{\mathcal{F}}(P, S)$, the $(P, S)$-biset $\Omega_{P}$ obtained by restricting the right action from $S$ to $P$ and the $(P, S)$-biset $\Omega_{\varphi}$ obtained by restricting the right action from $S$ to $P$ via $\varphi$ are isomorphic as $(P, S)-$ bisets.

(c) $|\Omega| /|S|=1 \bmod p$.

We call such an $(S, S)$-biset an $\mathcal{F}$-stable $(S, S)$-biset. Now let $(A, B): \mathcal{F} \rightarrow \mathcal{A}$ be a cohomological Mackey functor for $\mathcal{F}$ over the abelian category $\mathcal{A}$. For each transitive $(Q, R)$-biset $R \times_{\varphi} Q$ with $\varphi \in \operatorname{Hom}_{\mathcal{F}}(P, R), P \leq Q \leq S, R \leq S$, we have the composition

$$
A(R) \stackrel{A(\varphi)}{\longrightarrow} A(P)=B(P) \stackrel{B\left(\iota_{P}^{Q}\right)}{\longrightarrow} B(Q)=A(Q) .
$$

For each $(Q, R)$-biset $\Omega$ with

$$
\Omega=\coprod R \times_{\varphi} Q
$$

we can define a map $A(\Omega): A(R) \rightarrow A(Q)$ by

$$
A(\Omega):=\sum B(\iota) \circ A(\varphi) .
$$


Lemma 2.6 Let $(A, B): \mathcal{F} \rightarrow \mathcal{A}$ be a cohomological Mackey functor. Then:

(1) For each transitive $(Q, R)$-biset $R \times_{\varphi} Q$ the morphism (2-1) depends only on the isomorphism class of $R \times{ }_{\varphi} Q$ as $(Q, R)$-biset.

(2) For any $(Q, R)$-biset $\Omega$ the morphism (2-2) depends only on the isomorphism class of $\Omega$ as $(Q, R)-$ biset.

(3) For any $(Q, R)$-biset $\Omega$ and any monomorphism $\psi: P \rightarrow Q$ we have

$$
A(\psi) \circ A(\Omega)=A\left(\Omega_{\psi}\right),
$$

where $\Omega_{\psi}$ is the $(P, R)$-biset obtained by restricting the right action of $\Omega$ from $Q$ to $P$ via $\psi$.

(4) If $\mathcal{A}=\mathbb{Z}_{(p)}-\bmod$ and $\Omega$ is an $\mathcal{F}$-stable $(S, S)$-biset then

$$
A(S)^{\mathcal{F}}=\operatorname{Im}(A(\Omega): A(S) \rightarrow A(S)) .
$$

\section{Proof of Lemma 2.6}

Proof of (1) The transitive $(Q, R)$-bisets $R \times_{\varphi_{1}} Q$ and $R \times_{\varphi_{2}} Q$ with $\varphi_{1}: P_{1} \rightarrow R$, $\varphi_{2}: P_{2} \rightarrow R, P_{1}, P_{2} \leq Q$ are isomorphic as $(Q, R)$-bisets if and only if there exist elements $q \in Q$ and $r \in R$ such that the following diagram commutes:

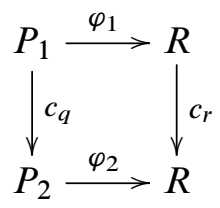

Hence both squares in the following diagram commute:

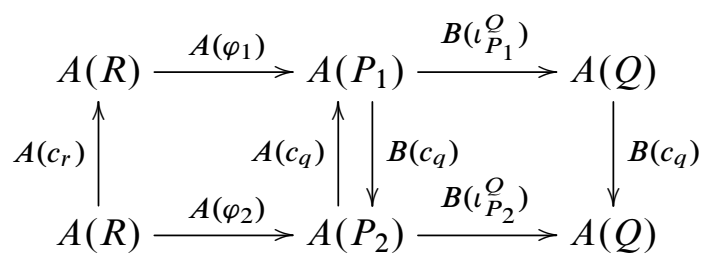

Using Properties (1) and (2) from Definition 2.2 one finds that

$$
B\left(\iota \iota_{P_{1}}^{Q}\right) \circ A\left(\varphi_{1}\right)=B\left(\iota_{P_{2}}^{Q}\right) \circ A\left(\varphi_{2}\right) .
$$

Proof of (2) Any automorphism of $\Omega$ permutes its transitive components via isomorphisms. So we may apply Lemma 2.6(1) to each component. 
Proof of (3) Write $\Omega$ as a disjoint union of transitive $(Q, R)$-bisets $\Omega=\coprod R \times{ }_{\varphi} Q$. The transitive $(Q, R)$ biset $R \times{ }_{\varphi} Q$ with $\varphi: K \rightarrow R, K \leq Q$ decomposes as a $(P, R)$-biset with $P$ acting via $\psi$ as follows:

$$
R \times \times_{\varphi} Q=\bigcup_{\substack{q \in \\ \psi(P) \backslash Q / K}} R \times \times_{\varphi \circ c_{q^{-1}} \circ \psi} P
$$

with $P \geq P \cap \psi^{-1}\left({ }^{q} K\right) \stackrel{\psi_{l}}{\rightarrow} \psi(P) \cap{ }^{q} K \stackrel{c_{q}-1}{\rightarrow} K \stackrel{\varphi}{\rightarrow} R$. Hence,

$$
A\left(\Omega_{\psi}\right)=\sum_{\varphi} \sum_{\substack{q \in \\ \psi(P) \backslash Q / K}} B\left(\iota_{P \cap \psi^{-1}\left({ }^{q} K\right)}^{P}\right) \circ A\left(\varphi \circ c_{q^{-1}} \circ \psi_{\mid}\right) .
$$

Using functoriality of $A$ and $B$ we get

$$
A\left(\Omega_{\psi}\right)=A(\tilde{\psi}) \circ\left(\sum_{\varphi} \sum_{\substack{q \in \\ \psi(P) \backslash Q / K}} B\left(\iota_{\psi(P) \cap^{q} K}^{\psi(P)}\right) \circ A\left(c_{q^{-1}}\right) \circ A(\varphi)\right),
$$

with $\tilde{\psi}: P \stackrel{\cong}{\rightarrow} \psi(P)$. Now the Mackey decomposition (3) from Definition 2.2 gives

$$
A\left(\Omega_{\psi}\right)=\sum_{\varphi} A(\tilde{\psi}) \circ A\left(\iota_{\psi(P)}^{Q}\right) \circ B\left(\iota_{K}^{Q}\right) \circ A(\varphi)=A(\psi) \circ A(\Omega) .
$$

Proof of (4) Let $z \in A(S)$. We want to see that $A(\Omega)(z) \in A(S)^{\mathcal{F}}$. So let $\psi$ be a morphism in $\operatorname{Hom}_{\mathcal{F}}(P, S)$. Then

$$
A(\psi)(A(\Omega)(z))=(A(\psi) \circ A(\Omega))(z)=A\left(\Omega_{\psi}\right)(z)
$$

by Part (3). By Proposition 2.5(b), the $(P, S)$-bisets $\Omega_{\psi}$ and $\Omega_{\iota_{P}}=\Omega_{P}$ are isomorphic as $(P, S)$-bisets. Then by Part (2) we have $A\left(\Omega_{\psi}\right)=A\left(\Omega_{l_{P}^{S}}\right)$. Hence,

$$
A(\psi)(A(\Omega)(z))=A\left(\Omega_{\psi}\right)(z)=A\left(\Omega_{\iota_{P}^{S}}\right)(z)=A\left(\iota_{P}^{S}\right)(A(\Omega)(z))
$$

by Part (3). Thus $A(\Omega)(z) \in A(S)^{\mathcal{F}}$.

Now let $z \in \mathcal{A}(S)^{\mathcal{F}}$. Then

$$
A(\Omega)(z)=\sum B(\iota)(A(\varphi)(z))=\sum B(\iota)(A(\iota)(z))
$$

as $z$ is $\mathcal{F}$-stable. Now by (4) of Definition 2.2 we get

$$
A(\Omega)(z)=\left(\sum|S: P|\right) \cdot z
$$

and by Proposition 2.5(c) the number $q=\left(\sum|S: P|\right)=|\Omega| /|S|$ is a $p^{\prime}$-number. So $A(\Omega)\left(\frac{z}{q}\right)=z$ and hence $z \in \operatorname{Im} A(\Omega)$. 
For a fusion system $\mathcal{F}$ over the $p$-group $S$ denote by $\operatorname{CohMack}_{\mathbb{Z}_{(p)}}(\mathcal{F})$ the abelian category with objects the cohomological Mackey functors with values in $\mathbb{Z}_{(p)}-\bmod$ and morphisms the natural transformations commuting with both the contravariant and covariant parts. This means that if $(A, B)$ and $\left(A^{\prime}, B^{\prime}\right)$ are cohomological Mackey functors, a morphism $\eta$ between them consists of a morphism of $\mathbb{Z}_{(p)}$-modules $\eta_{P}: A(P) \rightarrow A^{\prime}(P)$ for each $P \leq S$ such that for $\varphi \in \operatorname{Hom}_{\mathcal{F}}(P, Q)$ we have

$$
A^{\prime}(\varphi) \circ \eta_{Q}=\eta_{P} \circ A(\varphi) \text { and } \eta_{Q} \circ B(\varphi)=B^{\prime}(\varphi) \circ \eta_{P} .
$$

Lemma 2.7 Let $\mathcal{F}$ be a fusion system over the $p$-group $S$. Then the functor

$$
\operatorname{CohMack}_{\mathbb{Z}_{(p)}}(\mathcal{F}) \stackrel{(-)^{\mathcal{F}}}{\longrightarrow} \mathbb{Z}_{(p)}-\bmod
$$

sending $(A, B) \mapsto A^{\mathcal{F}}$ is exact.

Proof Let

$$
0 \Rightarrow\left(A_{1}, B_{1}\right) \Rightarrow\left(A_{2}, B_{2}\right) \stackrel{\eta}{\Rightarrow}\left(A_{3}, B_{3}\right) \Rightarrow 0
$$

be an exact sequence in $\operatorname{CohMack}_{\mathbb{Z}_{(p)}}(\mathcal{F})$. We want to prove that

$$
0 \rightarrow A_{1}^{\mathcal{F}} \longrightarrow A_{2}^{\mathcal{F}} \stackrel{\eta^{\mathcal{F}}}{\longrightarrow} A_{3}^{\mathcal{F}} \longrightarrow 0
$$

is exact in $\mathbb{Z}_{(p)}$-mod. The nontrivial assertion to prove is that the arrow $A_{2}^{\mathcal{F}} \rightarrow A_{3}^{\mathcal{F}}$ is an epimorphism. So let $z$ be an $\mathcal{F}$-stable element in $A_{3}(S)$. Fix an $(S, S)$-biset $\Omega$ satisfying the properties of Proposition 2.5. By Lemma 2.6 (4) there exists an element $z^{\prime} \in A_{3}(S)$ with $z=A_{3}(\Omega)\left(z^{\prime}\right)$. By hypothesis, the map

$$
A_{2}(S) \stackrel{\eta_{S}}{\longrightarrow} A_{3}(S)
$$

is an epimorphism and hence there exists an element $y^{\prime} \in A_{2}(S)$ with $\eta_{S}\left(y^{\prime}\right)=z^{\prime}$. By Lemma 2.6 (4) again we have that

$$
y \stackrel{\text { def }}{=} A_{2}(\Omega)\left(y^{\prime}\right)
$$

belongs to $A_{2}^{\mathcal{F}}$. Because $\eta$ commutes with the covariant and contravariant parts of $\left(A_{2}, B_{2}\right)$ and $\left(A_{3}, B_{3}\right)$, it is easy to see that

$$
\eta^{\mathcal{F}}(y)=\eta^{\mathcal{F}}\left(A_{2}(\Omega)\left(y^{\prime}\right)\right)=A_{3}(\Omega)\left(\eta^{\mathcal{F}}\left(y^{\prime}\right)\right)=A_{3}(\Omega)\left(z^{\prime}\right)=z .
$$

Proposition 2.8 Let $\mathcal{F}$ be a fusion system over $S$ and let $(A, B): \mathcal{F} \rightarrow \operatorname{CCh}\left(\mathbb{Z}_{(p)}\right)$ be a cohomological Mackey functor. Then

$$
H^{*}\left(A(S)^{\mathcal{F}}\right) \cong H^{*}(A(S))^{\mathcal{F}} .
$$

Proof This is a consequence of Lemma 2.7 and of the well-known fact that cohomology commutes with exact functors. 
Remark 2.9 Let $\mathcal{F}$ be a fusion system over the $p$-group $S$ and let $M$ be a trivial $\mathbb{Z}_{(p)} S$-module. By [3, Section 5] the cohomology of $\mathcal{F}$ is defined as

$$
H^{*}(\mathcal{F} ; M)=H^{*}(S ; M)^{\mathcal{F}},
$$

where $H^{*}(\cdot ; M): \mathcal{F} \rightarrow \mathbb{Z}_{(p)}$-modules is the cohomological Mackey functor with values $H^{*}(P ; M)$. If one could choose cochains $C^{*}(\cdot ; M): \mathcal{F} \rightarrow \operatorname{CCh}\left(\mathbb{Z}_{(p)}\right)$ such that $C^{*}(\cdot ; M)$ was the contravariant part of a cohomological Mackey functor then Proposition 2.8 would give the computational-purposes formula

$$
H^{*}(\mathcal{F} ; M)=H^{*}\left(C^{*}(S ; M)^{\mathcal{F}}\right) .
$$

In the next section some problems related to the functoriality of cochains will become apparent.

\section{A Mackey functor}

Let $\mathcal{F}$ be a fusion system over the $p$-group $S, T$ a strongly $\mathcal{F}$-closed subgroup of $S$ and $M$ a $\mathbb{Z}_{(p)}$-module with trivial $S$-action. In this section we prove that for every $n, m \geq 0$ the functor $H^{n, m}: \mathcal{F} \rightarrow \mathbb{Z}_{(p)}$-mod sending the subgroup $P \leq S$ to $H^{n}\left(P / P \cap T ; H^{m}(P \cap T ; M)\right)$ is the contravariant part of a cohomological Mackey functor $\mathcal{F} \rightarrow \mathrm{CCh}^{2}\left(\mathbb{Z}_{(p)}\right)$ with values in double (cochain) complexes (Definition 2.2). Here, by double complexes we mean the abelian category with objects families of $\mathbb{Z}_{(p)}$-modules $\left\{A^{n, m}\right\}_{n, m \in \mathbb{Z}}$ together with maps $d^{h}$ (horizontal differential) and $d^{v}$ (vertical differential)

$$
d^{h}: A^{n, m} \rightarrow A^{n+1, m} \quad \text { and } \quad d^{v}: A^{n, m} \rightarrow A^{n, m+1},
$$

such that $d^{h} d^{h}=d^{v} d^{v}=d^{h} d^{v}+d^{v} d^{h}=0$. A morphism from $\left\{A^{n, m}\right\}_{n, m \in \mathbb{Z}}$ to $\left\{A^{\prime n, m}\right\}_{n, m \in \mathbb{Z}}$ is a family of maps of $\mathbb{Z}_{(p)}$-modules $\left\{A^{n, m} \rightarrow A^{\prime n, m}\right\}_{n, m \in \mathbb{Z}}$ that commute with horizontal and vertical differentials.

For $P \leq S$ denote by $\bar{P}$ the group $P / P \cap T$. The bar resolutions $\mathcal{B}_{P}^{*}$ and $\mathcal{B}_{\bar{P}}^{*}$ for $P$ and $\bar{P}$ respectively are projective resolutions of the trivial module $\mathbb{Z}_{(p)}$ over $\mathbb{Z}_{(p)} P$ and $\mathbb{Z}_{(p)} \bar{P}$ respectively. Recall that the bar resolution is functorial (covariant) over finite groups and homomorphisms. Define $A^{*, *}(P)$ as the double complex associated to the short exact sequence

$$
0 \rightarrow P \cap T \rightarrow P \rightarrow \bar{P} \cong P T / T \rightarrow 0 .
$$

More precisely, for $n \geq 0$ and $m \geq 0$, we define

$$
A^{n, m}(P)=\operatorname{Hom}_{P}\left(\mathcal{B}_{\bar{P}}^{n} \otimes \mathcal{B}_{P}^{m}, M\right),
$$


where $P$ acts on $\mathcal{B}_{\bar{P}}^{n} \otimes \mathcal{B}_{P}^{m}$ by $p(y \otimes x)=\bar{p} y \otimes p x$ for $y \in \mathcal{B}_{\bar{P}}^{n}$ and $x \in \mathcal{B}_{P}^{m}$.

As the action of $P$ on $M$ is trivial the cochains in $A^{n, m}(P)$ are the homomorphisms $f \in \operatorname{Hom}\left(\mathcal{B}_{\bar{P}}^{n} \otimes \mathcal{B}_{P}^{m}, M\right)$ such that

$$
f(\bar{p} y \otimes p x)=f(y \otimes x)
$$

for all $y \in \mathcal{B}_{\bar{P}}^{n}, x \in \mathcal{B}_{P}^{m}$ and $p \in P$.

To obtain a double complex we consider the following horizontal and vertical differentials for $f \in A^{n, m}(P)$ :

$$
\begin{array}{ll}
d^{h}(f)(y \otimes x)=(-1)^{n+m+1} f(d(y) \otimes x), & y \in \mathcal{B}_{\bar{P}}^{n+1}, x \in \mathcal{B}_{P}^{m}, \\
d^{v}(f)(y \otimes x)=(-1)^{m+1} f(y \otimes d(x)), & y \in \mathcal{B}_{\bar{P}}^{n}, x \in \mathcal{B}_{P}^{m+1},
\end{array}
$$

where we are using the differential $d$ of the complexes $\mathcal{B}_{\bar{P}}^{*}$ and $\mathcal{B}_{P}^{*}$. We choose the signs as given by Mac Lane [13, XI.10.1] to ensure that $d^{h} d^{v}+d^{v} d^{h}=0$. We will obtain the functor $H^{n, m}$ by taking vertical cohomology followed by horizontal cohomology in $A^{n, m}$.

To define $A$ on morphisms notice that any morphism $\varphi \in \operatorname{Hom}_{\mathcal{F}}(P, Q)$ takes $P \cap T$ to $Q \cap T$ as $T$ is strongly $\mathcal{F}$-closed. Hence it induces a homomorphism

$$
\bar{\varphi}: \bar{P} \rightarrow \bar{Q} \text {. }
$$

Thus for any $\varphi \in \operatorname{Hom}_{\mathcal{F}}(P, Q)$ we may define

$$
A^{n, m}(Q) \stackrel{A^{n, m}(\varphi)}{\longrightarrow} A^{n, m}(P)
$$

mapping the cochain $f \in A^{n, m}(Q)$ to the cochain in $A^{n, m}(P)$ that takes $y \in \mathcal{B}_{\bar{P}}^{n}$ and $x \in \mathcal{B}_{P}^{m}$ to

$$
f\left(\mathcal{B}^{n}(\bar{\varphi})(y) \otimes \mathcal{B}^{m}(\varphi)(x)\right),
$$

where $\mathcal{B}^{n}(\bar{\varphi})$ and $\mathcal{B}^{m}(\varphi)$ are the usual morphisms between bar resolutions. They commute with differentials and satisfy

$$
\mathcal{B}^{n}(\bar{\varphi})(\bar{p} \cdot y)=\bar{\varphi}(\bar{p}) \cdot \mathcal{B}^{n}(\bar{\varphi})(y)
$$

for every $y \in \mathcal{B}_{\bar{P}}^{n}$ and every $\bar{p} \in \bar{P}$ and

$$
\mathcal{B}^{m}(\varphi)(p \cdot x)=\varphi(p) \cdot \mathcal{B}^{m}(\varphi)(x)
$$

for every $x \in \mathcal{B}_{P}^{m}$ and $p \in P$. It is straightforward that $A^{n, m}(\varphi)(f) \in A^{n, m}(P)$ and that the family of morphisms $\left\{A^{n, m}(\varphi)\right\}_{n, m \geq 0}$ commutes with the horizontal and vertical differentials of the double complexes $A^{*, *}(Q)$ and $A^{*, *}(P)$. 
Remark 3.1 By definition the fusion system $\mathcal{F} / T$ is defined over the $p$-group $S / T$. For $T \leq P, Q \leq S$ the morphisms in $\operatorname{Hom}_{\mathcal{F} / T}(P / T, Q / T)$ are those homomorphisms $\bar{\psi}: P / T \rightarrow Q / T$ induced on the quotient from $\psi \in \operatorname{Hom}_{\mathcal{F}}(P, Q)$.

For $P, Q \leq S$ and $\varphi \in \operatorname{Hom}_{\mathcal{F}}(P, Q)$ we have a morphism $\bar{\varphi}: \bar{P} \rightarrow \bar{Q}$. Then we have a commutative diagram

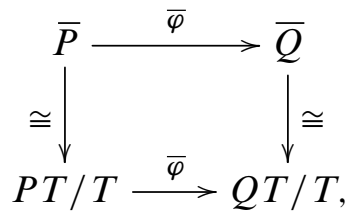

where the $\bar{\varphi}$ are induced by $\varphi$ and where the vertical arrows are the natural isomorphisms. According to [7, 5.10] bottom morphism $\bar{\varphi}$ belongs to $\mathcal{F} / T$, ie, there exists $\psi \in \operatorname{Hom}_{\mathcal{F}}(P T, Q T)$ such that the induced map $\bar{\psi}: P T / T \rightarrow Q T / T$ coincides with the given one.

Remark 3.2 The construction of $A^{n, m}$ is clearly functorial and hence so far we have a contravariant functor $A^{*, *}: \mathcal{F} \rightarrow \mathrm{CCh}^{2}\left(\mathbb{Z}_{(p)}\right)$ with values in double complexes.

Now we define $B^{n, m}(P)=A^{n, m}(P)$ for every $P \leq S$ and $n, m \geq 0$. For each morphism $\varphi \in \operatorname{Hom}_{\mathcal{F}}(P, Q)$ we will define a morphism of double complexes

$$
B^{n, m}(\varphi): A^{n, m}(P) \rightarrow A^{n, m}(Q) .
$$

This will not make $B$ into a covariant functor $\mathcal{F} \rightarrow \mathrm{CCh}^{2}\left(\mathbb{Z}_{(p)}\right)$ as the definition depends on a choice of representatives. Nevertheless, $B$ will become functorial once we pass to cohomology.

To define $B^{*, *}(\psi)$ on $\psi \in \operatorname{Hom}_{\mathcal{F}}(P, Q)$, write $\psi=\iota \circ \tilde{\psi}$, where $\tilde{\psi}: P \rightarrow \psi(P)$ is an isomorphism and $\iota$ is the inclusion $\psi(P) \leq Q$, and set

$$
B^{*, *}(\psi)=B^{*, *}(\iota) \circ A^{*, *}\left(\tilde{\psi}^{-1}\right) .
$$

So we just need to define $B$ on inclusions.

So let $\iota$ be the inclusion between subgroups $P \leq Q$ of $S$. There are maps of $\mathbb{Z}_{(p)} P-$ chain complexes and of $\mathbb{Z}_{(p)} \bar{P}$-chain complexes respectively

$$
\tau_{*}^{Q, P}: \mathcal{B}_{Q}^{*} \rightarrow \mathcal{B}_{P}^{*}, \quad \bar{\tau}_{*}^{Q, P}: \mathcal{B}_{\bar{Q}}^{*} \rightarrow \mathcal{B}_{\bar{P}}^{*},
$$

built as in [4, (D), page 82]. More precisely, the map $\tau_{*}^{Q, P}$ is induced by a map of left $P$-sets $Q \stackrel{\rho}{\rightarrow} P$ defined as follows: fix a set of representatives for the right cosets $P \backslash Q$, then $\rho(q)=q \bar{q}^{-1}$, where $\bar{q}$ is the representative with $P q=P \bar{q}$. The map 
$\bar{\tau}_{*}^{Q, P}$ is defined analogously choosing representatives for the right cosets $\bar{P} \backslash \bar{Q}$. These choices of representatives prevent $B^{p, q}$ from being functorial.

We define the map

$$
\begin{array}{r}
B^{n, m}(\iota): \operatorname{Hom}_{P}\left(\mathcal{B}_{\bar{P}}^{n} \otimes \mathcal{B}_{P}^{m}, M\right) \rightarrow \operatorname{Hom}_{Q}\left(\mathcal{B}_{\bar{Q}}^{n} \otimes \mathcal{B}_{Q}^{m}, M\right), \\
B^{n, m}(\iota)(f)(y \otimes x)=\sum_{w \in Q / P} f\left(\bar{\tau}_{n}^{Q, P}\left(\bar{w}^{-1} y\right) \otimes \tau_{m}^{Q, P}\left(w^{-1} x\right)\right),
\end{array}
$$

where $w$ runs over a set of representatives of the left cosets $Q / P$. This formula can be thought as a relative transfer formula for twisted coefficients. Clearly its definition does not depend on the representatives $w$ chosen and $B^{n, m}(\iota)(f) \in A^{n, m}(Q)$. Moreover, $B^{n, m}(\iota)$ commutes with both the horizontal and vertical differentials as $\tau_{*}$ and $\bar{\tau}_{*}$ do and so it is a map of double complexes.

Remark 3.3 By Park [14] there are finite groups $G$ and $\bar{G}$ such that $S$ is a $p$ subgroup of $G$ (not necessarily a Sylow $p$-subgroup), $\bar{S}=S / T$ is a $p$-subgroup of $\bar{G}$ (not necessarily a Sylow $p$-subgroup) and with $\mathcal{F}=\mathcal{F}_{S}(G)$ and $\mathcal{F} / T=\mathcal{F}_{\bar{S}}(\bar{G})$. Let $B_{G}^{*}$ and $B_{\bar{G}}^{*}$ be the bar resolutions of $G$ and $\bar{G}$ respectively. Then we could have defined for $P \leq S$

$$
A^{n, m}(P)=\operatorname{Hom}_{P}\left(\mathcal{B}_{\bar{G}}^{n} \otimes \mathcal{B}_{G}^{m}, M\right),
$$

where $P$ acts on $\mathcal{B}_{\bar{G}}^{n} \otimes \mathcal{B}_{G}^{m}$ by restricting the actions of $G$ on $\mathcal{B}_{G}^{*}$ and of $\bar{G}$ on $\mathcal{B}_{\bar{G}}^{*}$. This means that $p(y \otimes x)=\bar{p} y \otimes p x$ for $p \in P$. In this setup clearly one can define a functorial $B^{n, m}$ on inclusions. On the other hand, to realize a morphism $\varphi: P \rightarrow Q$ we need to choose $g \in N_{G}(P, Q)$ with $\varphi=c_{g}$ and $\bar{g} \in N_{\bar{G}}(\bar{P}, \bar{Q})$ with $\bar{\varphi}=c_{\bar{g}}$ and then define

$$
A^{n, m}(\varphi)(y \otimes x)=(\bar{g} y \otimes g x) .
$$

It is clear that in general $A^{n, m}$ defined this way will not be functorial on morphisms. If one could choose $A^{n, m}$ and $B^{n, m}$ such that $\left(A^{n, m}, B^{n, m}\right): \mathcal{F} \rightarrow \mathrm{CCh}^{2}\left(\mathbb{Z}_{(p)}\right)$ was a Mackey functor then the proof of Theorem 4.1 would be simpler.

On each double complex $A^{*, *}(P)$ with $P \leq S$ we may take vertical cohomology followed by horizontal cohomology to obtain $H^{*}\left(\bar{P} ; H^{*}(P \cap T ; M)\right)$ [13, Equation (10.2), page 352]. For any homomorphism $\varphi \in \operatorname{Hom}_{\mathcal{F}}(P, Q)$ the maps $A^{*, *}(\varphi)$ and $B^{*, *}(\varphi)$ are maps of double complexes and hence they induce maps

$$
\begin{aligned}
& H^{n, m}(A)(\varphi): H^{n}\left(\bar{Q} ; H^{m}(Q \cap T ; M)\right) \rightarrow H^{n}\left(\bar{P} ; H^{m}(P \cap T ; M)\right), \\
& H^{n, m}(B)(\varphi): H^{n}\left(\bar{P} ; H^{m}(P \cap T ; M)\right) \rightarrow H^{n}\left(\bar{Q} ; H^{m}(Q \cap T ; M)\right) .
\end{aligned}
$$


Lemma 3.4 For $\varphi: P \rightarrow Q$ the map $H^{n, m}(A)(\varphi)$ factors as

$$
\begin{aligned}
H^{n}\left(\bar{Q} ; H^{m}(Q \cap T ; M)\right) \stackrel{H^{n}(\bar{\varphi})}{\longrightarrow} H^{n}\left(\bar{P} ; H^{m}(Q \cap T ; M)\right) & \\
& \stackrel{H^{m}(\varphi)}{\longrightarrow} H^{n}\left(\bar{P} ; H^{m}(P \cap T ; M)\right),
\end{aligned}
$$

where

- $H^{n}(\bar{\varphi})$ is the map induced by $\bar{\varphi}$ in cohomology with $H^{m}(Q \cap T ; M)$-coefficients,

- $H^{m}(\varphi)$ is the map induced by the change of coefficients

$$
H^{m}(\varphi): H^{m}(Q \cap T ; M) \rightarrow H^{m}(P \cap T ; M) .
$$

This map is a map of $\mathbb{Z}_{(p)} \bar{P}$-modules where $\bar{P}$ acts on $H^{m}(Q \cap T ; M)$ via $\bar{P} \stackrel{\bar{\varphi}}{\rightarrow}$ $\overline{\varphi(P)} \leq \bar{Q}$.

Proof By construction.

Lemma 3.5 If $P \leq Q$ and $\iota$ denotes the inclusion then the map $H^{n, m}(B)(\iota)$ factors as

$$
\begin{aligned}
& H^{n}\left(\bar{P} ; H^{m}(P \cap T ; M)\right) \stackrel{H^{m}\left(\mathrm{tr}^{\prime}\right)}{\longrightarrow} H^{n}\left(\bar{P} ; H^{m}(Q \cap T ; M)\right) \\
& \stackrel{H^{n}(\mathrm{tr})}{\longrightarrow} H^{n}\left(\bar{Q} ; H^{m}(Q \cap T ; M)\right),
\end{aligned}
$$

where

- $H^{n}(\operatorname{tr})$ is the transfer map in cohomology with $H^{m}(Q \cap T ; M)$-coefficients,

- $H^{m}\left(\mathrm{tr}^{\prime}\right)$ is the map induced by the change of coefficients given by the transfer map in cohomology:

$$
H^{m}\left(\operatorname{tr}^{\prime}\right): H^{m}(P \cap T ; M) \rightarrow H^{m}(Q \cap T ; M)
$$

This map is a map of $\mathbb{Z}_{(p)} \bar{P}$-modules where $\bar{P}$ acts on $H^{m}(Q \cap T ; M)$ via $\bar{P} \leq \bar{Q}$.

Proof Choose representatives $z_{i} \in \bar{Q}$ of the left cosets $\bar{Q} / \bar{P}$ and representatives $t_{j} \in Q \cap T$ of the left cosets $(Q \cap T) /(P \cap T)$. Choose also representatives $q_{k} \in Q$ of the left cosets $\bar{Q}=Q /(Q \cap T)$. Then each $z_{i} \in \bar{Q}$ is represented as $z_{i}=\overline{q_{k_{i}}}$ for a unique $k_{i}$. It is an exercise to prove that the set of elements of $Q q_{k_{i}} t_{j}$ for all $i$ and $j$ is a set of representatives of $Q / P$. Then we can rewrite Equation (3-2) as

$$
\sum_{z_{i} \in \bar{Q} / \bar{P}} \sum_{t_{j} \in(Q \cap T) /(P \cap T)} f\left(\bar{\tau}_{n}^{Q, P}\left({\overline{q_{k_{i}} t_{j}}}^{-1} y\right) \otimes \tau_{m}^{Q, P}\left(\left(q_{k_{i}} t_{j}\right)^{-1} x\right)\right),
$$


Because $t_{j} \in Q \cap T$ then $\overline{q_{k_{i}} t_{j}}=\overline{q_{k_{i}}}$ and the formula simplifies to

$$
\sum_{z_{i} \in \bar{Q} / \bar{P}} \sum_{t_{j} \in(Q \cap T) /(P \cap T)} f\left(\bar{\tau}_{n}^{Q, P}\left(\bar{q}_{k_{i}}-1 y\right) \otimes \tau_{m}^{Q, P}\left(t_{j}^{-1} q_{k_{i}}{ }^{-1} x\right)\right) .
$$

This coincides with the composition in the statement of the lemma.

Lemma 3.5 proves in particular that the definition of $H^{n, m}(B)(\iota)$ does not depend on the representatives chosen to construct the maps $\tau_{*}^{Q, P}$ and $\bar{\tau}_{*}^{Q, P}$. (Although $B^{n, m}(\iota)$ do depends on them.)

Corollary 3.6 For $n, m \geq 0$ the assignment

$$
H^{n, m}(B): \mathcal{F} \rightarrow \mathbb{Z}_{(p)}-\bmod
$$

taking $P$ to $H^{n}\left(\bar{P} ; H^{m}(P \cap T ; M)\right)$ and taking $\varphi \in \operatorname{Hom}_{\mathcal{F}}(P, Q)$ to $H^{n, m}(B)(\varphi)$ is a functor.

Proof By Remarks 2.4 and 3.2 and Equation (3-1) it is enough to prove that for any $P \leq Q \leq R$ we have

$$
H^{n, m}(B)\left(\iota_{Q}^{R}\right) \circ H^{n, m}(B)\left(\iota_{P}^{Q}\right)=H^{n, m}(B)\left(\iota_{P}^{R}\right)
$$

and for any $P \leq Q \stackrel{\varphi}{\rightarrow} \varphi(Q)$ we have

$$
H^{n, m}(B)\left(\iota_{P}^{Q}\right) \circ H^{n, m}(A)\left(\varphi_{\mid P}\right)=H^{n, m}(A)(\varphi) \circ H^{n, m}(B)\left(\iota_{\varphi(P)}^{\varphi(Q)}\right) .
$$

We can check both conditions at the level of cochains: For the first condition, the definitions (3-2) of $B^{n, m}\left(\iota_{P}^{Q}\right), B^{n, m}\left(\iota_{Q}^{R}\right)$ and $B^{n, m}\left(\iota_{P}^{R}\right)$ depend upon choices of representatives for the right cosets

$$
P \backslash Q \text { and } \bar{P} \backslash \bar{Q}, \quad Q \backslash R \text { and } \bar{Q} \backslash \bar{R}, \quad P \backslash R \text { and } \bar{P} \backslash \bar{R}
$$

respectively. Fix choices of representatives for the first four right cosets. Then the bijections $P \backslash Q \times Q \backslash R \rightarrow P \backslash R$ and $\bar{P} \backslash \bar{Q} \times \bar{Q} \backslash \bar{R} \rightarrow \bar{P} \backslash \bar{R}$ provide choices for the last two right cosets. With these choices we have

$$
B^{n, m}\left(\iota_{Q}^{R}\right) \circ B^{n, m}\left(\iota_{P}^{Q}\right)=B^{n, m}\left(\iota_{P}^{R}\right) .
$$

For the second condition, the maps $B^{n, m}\left(\iota_{P}^{Q}\right)$ and $B^{n, m}\left(\iota_{\varphi(P)}^{\varphi(Q)}\right)$ depend on choices of representatives for the right cosets

$$
P \backslash Q \text { and } \bar{P} \backslash \bar{Q}, \quad \varphi(P) \backslash \varphi(Q) \text { and } \overline{\varphi(P)} \backslash \overline{\varphi(Q)}
$$


respectively. Fix representatives in $P \backslash Q$ and $\bar{P} \backslash \bar{Q}$ and force the other choices via the bijections

$$
P \backslash Q \stackrel{\varphi}{\rightarrow} \varphi(P) \backslash \varphi(Q) \quad \text { and } \quad \bar{P} \backslash \bar{Q} \stackrel{\bar{\varphi}}{\rightarrow} \overline{\varphi(P)} \backslash \overline{\varphi(Q)} .
$$

Then we have

$$
B^{n, m}\left(\iota_{P}^{Q}\right) \circ A^{n, m}\left(\varphi_{\mid P}\right)=A^{n, m}(A)(\varphi) \circ B^{n, m}\left(\iota_{\varphi(P)}^{\varphi(Q)}\right) .
$$

Proposition 3.7 For each $p, q \geq 0$ the functor $\mathcal{F} \rightarrow \mathbb{Z}_{(p)}-\bmod$ with values

$$
H^{p}\left(\bar{P} ; H^{q}(P \cap T ; M)\right.
$$

and taking $\varphi \in \operatorname{Hom}_{\mathcal{F}}(P, Q)$ to $H^{p, q}(A)(\varphi)$ is a cohomological Mackey functor with covariant part taking $\varphi \in \operatorname{Hom}_{\mathcal{F}}(P, Q)$ to $H^{p, q}(B)(\varphi)$.

Proof Property (1) from Definition 2.2 holds by Equation (3-1). Property (2) follows from Property (1), the well known fact that conjugation induces the identity on cohomology, from Lemma 3.4 and from $\overline{c_{p}}=c_{\bar{p}}$ for $p \in P \leq S$. Now we check Property (3), also known as the Mackey condition or double coset formula. So let $Q, R \leq P \leq S$. We will prove this condition at the level of cochains, ie

$$
A^{n, m}\left(\iota_{Q}^{P}\right) \circ B^{n, m}\left(\iota_{R}^{P}\right)=\sum_{x \in Q \backslash P / R} B^{n, m}\left(\iota_{Q \cap x_{R}}^{Q}\right) \circ A^{n, m}\left({ }^{\iota_{Q} R}{ }^{x}{ }^{x} R\right) \circ A^{n, m}\left(c_{x^{-1} \mid x_{R}}\right) .
$$

So let $f \in A^{n, m}(R)=\operatorname{Hom}_{R}\left(\mathcal{B}_{\bar{R}}^{n} \otimes \mathcal{B}_{R}^{m}, M\right), y \in \mathcal{B}_{\bar{Q}}^{n}$ and $x \in \mathcal{B}_{Q}^{m}$. Then

$$
\begin{aligned}
A^{n, m}\left(\iota_{Q}^{P}\right)\left(B^{n, m}\left(\iota_{R}^{P}\right)(f)\right)(y \otimes x) & =B^{n, m}\left(\iota_{R}^{P}\right)(f)\left(\overline{\iota_{Q}^{P}}(y) \otimes \iota_{Q}^{P}(x)\right) \\
& =B^{n, m}\left(\iota_{R}^{P}\right)(f)(y \otimes x) .
\end{aligned}
$$

This equals

$$
\sum_{w \in P / R} f\left(\bar{\tau}_{n}^{P, R}\left(\bar{w}^{-1} y\right) \otimes \tau_{m}^{P, R}\left(w^{-1} x\right)\right),
$$

where $w$ runs over a set of representatives of the left cosets $P / R, \bar{\tau}_{n}^{P, R}: \mathcal{B}_{\bar{P}}^{n} \rightarrow \mathcal{B}_{\bar{R}}^{n}$ and $\tau_{m}^{P, R}: \mathcal{B}_{P}^{m} \rightarrow \mathcal{B}_{R}^{m}$. Now we let $Q$ acts on the left on $P / R$ and we group together the terms corresponding to a given $Q$-orbit in $P / R$ :

$$
\sum_{p \in Q \backslash P / R} \sum_{q \in Q / Q \cap^{p} R} f\left(\bar{\tau}_{n}^{P, R}\left(\overline{q p}^{-1} y\right) \otimes \tau_{m}^{P, R}\left((q p)^{-1} x\right)\right),
$$

where now $p$ runs over a set of representatives for the double cosets $Q \backslash P / R$ and $q$ runs over a set of representatives of the left cosets $Q / Q \cap{ }^{n} R$. This equals

$$
\sum_{p \in Q \backslash P / R} \sum_{q \in Q / Q \cap^{p} R} f\left(\bar{\tau}_{n}^{P, R}\left(\bar{p}^{-1} \bar{q}^{-1} y\right) \otimes \tau_{m}^{P, R}\left(p^{-1} q^{-1} x\right)\right) .
$$


The right-hand side of the Mackey formula is

$$
\sum_{p \in Q \backslash P / R} \sum_{q \in Q / Q \cap^{p} R} f\left(\bar{p}^{-1} \bar{\tau}_{n}^{Q, Q \cap^{p} R}\left(\bar{q}^{-1} y\right) \bar{p} \otimes p^{-1} \tau_{q}^{Q, Q \cap^{p} R}\left(q^{-1} x\right) p\right)
$$

with

$$
\bar{\tau}_{n}^{Q, Q \cap{ }^{p} R}: \mathcal{B}_{\bar{Q}}^{n} \rightarrow \mathcal{B}_{\overline{Q \cap p} R}^{n}, \quad \tau_{m}^{Q, Q \cap^{p} R}: \mathcal{B}_{Q}^{m} \rightarrow \mathcal{B}_{Q \cap \cap^{p} R}^{m}
$$

and where we have realized $c_{p^{-1}{ }^{p} R}$ at the level of cochains as

$$
A^{n, m}\left(c_{p^{-1}{ }^{p} R}\right)(y \otimes x)=\left(\bar{p}^{-1} y \bar{p} \otimes p^{-1} x p\right) .
$$

The map $\tau_{m}^{P, R}$ depends on a choice of representatives for the right cosets $R \backslash P$. Similarly, for any representative $p \in Q \backslash P / R$, the map $\tau_{m}^{Q, Q \cap^{p} R}$ is built out of a set of representatives of $Q \cap{ }^{p} R \backslash Q$. We want to choose representatives of $R \backslash P$ and of $Q \cap{ }^{p} R \backslash Q$ for each double coset $Q p R$ such that

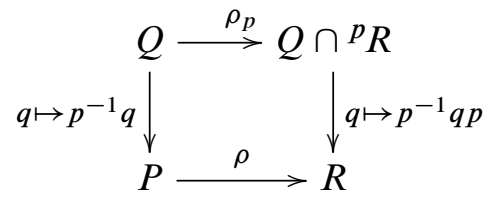

commutes for each double coset $Q p R$. For this is enough to choose arbitrary representatives $q$ of $Q \cap{ }^{p} R \backslash Q$ for each double coset $Q p R$ and build the representatives in $R \backslash P$ via the bijection

$$
\coprod_{p \in Q \backslash P / R} Q \cap{ }^{p} R \backslash Q \rightarrow R \backslash P
$$

that takes $\left(Q \cap{ }^{p} R\right) q$ to $R p^{-1} q$. The same argument for $\bar{\tau}_{n}^{P, R}$ and the maps $\bar{\tau}_{n}^{Q, Q \cap^{p} R}$ finishes the proof of Property (3).

To prove Property (4) we go back to the level of cohomology. Let $P \leq Q \leq S$. By Lemmas 3.4 and 3.5 the composition $H^{n, m}(B)\left(\iota_{P}^{Q}\right) \circ H^{n, m}(A)\left(\iota_{P}^{Q}\right)$ is equal to

$$
H^{n}(\operatorname{tr}) \circ H^{m}\left(\operatorname{tr}^{\prime}\right) \circ H^{m}\left(\iota_{P}^{Q}\right) \circ H^{n}\left(\iota_{P}^{Q}\right) .
$$

Because cohomology over finite groups is a cohomological Mackey functor we know that $H^{m}\left(\operatorname{tr}^{\prime}\right) \circ H^{m}\left(\iota_{P}^{Q}\right)$ is multiplication by $|Q \cap T| /|P \cap T|$. Moving out this factor we are left with

$$
H^{n}(\operatorname{tr}) \circ H^{n}\left(\iota_{P}^{\bar{Q}}\right) .
$$

As $\iota_{P}^{Q}=\iota \frac{\bar{Q}}{P}$ we obtain again by properties of cohomology for finite groups that this composition is multiplication by $|\bar{Q}| /|\bar{P}|$. So finally we obtain that

$$
H^{n, m}(B)\left(\iota_{P}^{Q}\right) \circ H^{p, q}(A)\left(\iota_{P}^{Q}\right)
$$


is multiplication by

$$
\frac{|Q \cap T|}{|P \cap T|} \frac{|\bar{Q}|}{|\bar{P}|}=\frac{|Q|}{|P|} .
$$

\section{Construction of the spectral sequence}

In this section we prove the main theorem of this paper:

Theorem 4.1 Let $\mathcal{F}$ be a fusion system over the $p$-group $S, T$ a strongly $\mathcal{F}$-closed subgroup of $S$ and $M$ a $\mathbb{Z}_{(p)}$-module with trivial $S$-action. Then there is a first quadrant cohomological spectral sequence with second page

$$
E_{2}^{n, m}=H^{n}\left(S / T ; H^{m}(T ; M)\right)^{\mathcal{F}}
$$

and converging to $H^{n+m}(\mathcal{F} ; M)$.

Proof For each subgroup $P \leq S$ we have the short exact sequence

$$
P \cap T \rightarrow P \rightarrow \bar{P}=P / P \cap T .
$$

The construction of the Lyndon-Hochschild-Serre spectral sequence in [13, XI.10.1] associates to this short exact sequence a double complex naturally isomorphic to the double complex

$$
A^{n, m}(P)=\operatorname{Hom}_{P}\left(\mathcal{B}_{\bar{P}}^{n} \otimes \mathcal{B}_{P}^{m}, M\right)
$$

defined in Section 3. This double complex we can filter either by columns or rows. If we filter by columns we obtain a spectral sequence $\left\{{ }^{c} E_{k}^{*, *}(P), d_{k}\right\}_{0 \leq k \leq \infty}$ whose second page is ${ }^{c} E_{2}^{n, m}(P)=H^{n}\left(\bar{P} ; H^{m}(P \cap T ; M)\right)$. If we filter by rows we obtain a spectral sequence $\left\{{ }^{r} E_{k}^{*, *}(P), d_{k}\right\}_{0 \leq k \leq \infty}$ whose second page collapses as ${ }^{r} E_{2}^{n, m}(P)=$ $H^{m}(P ; M)$ for $n=0$ and ${ }^{r} E_{2}^{n, m}(P)=0$ for $n>0$.

For each morphism $\varphi \in \operatorname{Hom}_{\mathcal{F}}(P, Q)$ we have morphisms of double complexes

$$
A^{n, m}(\varphi): A^{n, m}(Q) \rightarrow A^{n, m}(P) \quad \text { and } \quad B^{n, m}(\varphi): A^{n, m}(P) \rightarrow A^{n, m}(Q)
$$

defined in Section 3. These morphisms of double complexes induce morphisms of spectral sequences consisting of a sequence of morphisms of differential bigraded $\mathbb{Z}_{(p)}$-modules

$$
\begin{aligned}
& { }^{c} E_{k}^{*, *}(A)(\varphi):{ }^{c} E_{k}^{*, *}(Q) \rightarrow{ }^{c} E_{k}^{*, *}(P), \\
& { }^{c} E_{k}^{*, *}(B)(\varphi):{ }^{c} E_{k}^{*, *}(P) \rightarrow{ }^{c} E_{k}^{*, *}(Q), \\
& { }^{r} E_{k}^{*, *}(A)(\varphi):{ }^{r} E_{k}^{*, *}(Q) \rightarrow{ }^{r} E_{k}^{*, *}(P), \\
& { }^{r} E_{k}^{*, *}(B)(\varphi):{ }^{r} E_{k}^{*, *}(P) \rightarrow{ }^{r} E_{k}^{*, *}(Q),
\end{aligned}
$$


for $0 \leq k \leq \infty$. We deal now with the filtration by columns spectral sequences. The second page ${ }^{c} E_{2}^{*, *}$ is obtained by computing vertical cohomology followed by horizontal cohomology in the double complex $A^{*, *}$. Hence we have

$$
\begin{gathered}
{ }^{c} E_{2}^{n, m}(P)=H^{n}\left(\bar{P} ; H^{m}(P \cap T ; M)\right), \\
{ }^{c} E_{2}^{n, m}(A)(\varphi)=H^{n, m}(A)(\varphi), \\
{ }^{c} E_{2}^{n, m}(B)(\varphi)=H^{n, m}(B)(\varphi),
\end{gathered}
$$

for $P \leq S$ and $\varphi \in \operatorname{Hom}_{\mathcal{F}}(P, Q)$, where $H^{n, m}(A)$ and $H^{n, m}(B)$ are functors $\mathcal{F} \rightarrow$ $\mathbb{Z}_{(p)}$-mod by Remark 3.2 and Corollary 3.6 respectively. Hence, for each $2 \leq k \leq \infty$, we have a contravariant functor

$$
{ }^{c} E_{k}^{*, *}(A): \mathcal{F} \rightarrow \text { differential bigraded } \mathbb{Z}_{(p)} \text {-modules }
$$

and a covariant functor

$$
{ }^{c} E_{k}^{*, *}(B): \mathcal{F} \rightarrow \text { differential bigraded } \mathbb{Z}_{(p)} \text {-modules. }
$$

On the one hand, we can take invariants for each $2 \leq k \leq \infty$ to obtain a differential bigraded $\mathbb{Z}_{(p)}$-module

$$
{ }^{c} E_{k}^{*, * \mathcal{F}}=\left\{z \in{ }^{c} E_{k}^{*, *}(S) \mid{ }^{c} E_{k}^{*, *}(A)(\varphi)(z)={ }^{c} E_{k}^{*, *}(A)\left(\iota_{P}^{S}\right)(z) \text { for } P \stackrel{\varphi}{\rightarrow} S\right\} .
$$

On the other hand, for $k=2$, we have by Proposition 3.7 that $\left({ }^{c} E_{2}^{*, *}(A),{ }^{c} E_{2}^{*, *}(B)\right)$ is a cohomological Mackey functor. Because ${ }^{c} E_{k+1}^{*, *}=H^{*}\left({ }^{c} E_{k}^{*, *}, d_{k}\right)$ and because passing to cohomology preserves cohomological Mackey functors we deduce that ${ }^{c} E_{k}^{*}, *(A)$ is a cohomological Mackey functor with covariant part ${ }^{c} E_{k}^{*, *}(B)$ for $2 \leq k<\infty$. By Proposition 2.8 we obtain then that

$$
{ }^{c} E_{k+1}^{*, * \mathcal{F}}=H^{*}\left({ }^{c} E_{k}^{*, *}, d_{k}\right)^{\mathcal{F}}=H^{*}\left({ }^{c} E_{k}^{*, * \mathcal{F}}, d_{k}\right)
$$

for $2 \leq k<\infty$. Fix now $n \geq 0$ and $m \geq 0$. For each subgroup $P \leq S$ we have

$$
{ }^{c} E_{k}^{n, m}(P)={ }^{c} E_{k+1}^{n, m}(P)=\cdots={ }^{c} E_{\infty}^{n, m}(P)
$$

for $k$ big enough. Because there are a finite number of subgroups of $S$ we deduce that

$$
{ }^{c} E_{k}^{n, m \mathcal{F}}={ }^{c} E_{k+1}^{n, m \mathcal{F}}=\cdots={ }^{c} E_{\infty}^{n, m \mathcal{F}}
$$

for $k$ big enough. Hence Equation (4-1) also holds for $k=\infty$ and we have obtained a spectral sequence

$$
\left\{{ }^{c} E_{k}^{*, *} \mathcal{F}, d_{k}\right\}_{2 \leq k \leq \infty} .
$$


To study whether this spectral sequence converges recall that for $P \leq S$ the spectral sequence $\left\{{ }^{c} E_{k}^{*, *}(P), d_{k}\right\}_{0 \leq k \leq \infty}$ converges to $H^{*}(P ; M)$. Hence we have short exact sequences

$$
0 \longrightarrow F^{n} H^{n+m}(P ; M) \longrightarrow F^{n+1} H^{n+m}(P ; M) \longrightarrow{ }^{c} E_{\infty}^{n, m}(P) \longrightarrow 0,
$$

where

$$
0 \subseteq \cdots \subseteq F^{n} H^{n+m}(P ; M) \subseteq F^{n+1} H^{n+m}(P ; M) \subseteq \cdots \subseteq H^{n+m}(P ; M)
$$

is the filtration induced on $H^{*}(P ; M)$ by the filtration by columns on the double complex $A^{*, *}(P)$. This short exact sequence is natural with respect to morphisms of double complexes. Hence for each $\varphi \in \operatorname{Hom}_{\mathcal{F}}(P, Q)$ we have morphisms of short exact sequences

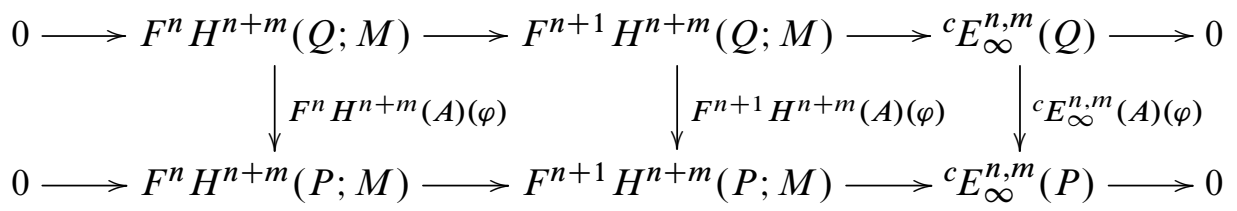

and

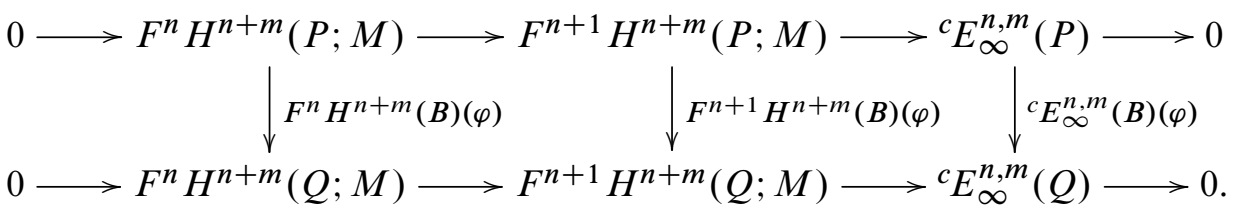

We want to show that the morphism $H^{n}(A)(\varphi): H^{n}(Q ; M) \rightarrow H^{n}(P ; M)$ and the morphism $H^{n}(B)(\varphi): H^{n}(P ; M) \rightarrow H^{n}(Q ; M)$ induced by $A$ and $B$ on the targets of the spectral sequences are the usual maps in cohomology of groups. We consider the total complex of the double complex $A^{*, *}(P)$ defined as usual by

$$
\operatorname{Tot}^{s}(A)=\bigoplus_{n+m=s} A^{n, m}(P)
$$

and with total differential $d^{h}+d^{v}$. There is a chain map given by

$$
\zeta: \operatorname{Hom}_{P}\left(\mathcal{B}_{P}^{*}, M\right) \rightarrow \operatorname{Tot}^{*}(A)
$$

sending $f \in \operatorname{Hom}_{P}\left(\mathcal{B}_{P}^{m}, M\right)$ to $\zeta(f) \in A^{0, m}$ defined by

$$
\zeta(f)(\bar{p} \otimes x)=f(x), \quad \bar{p} \in \bar{P}, x \in \mathcal{B}_{P}^{m} .
$$


The map $\zeta$ induces an isomorphism between the cohomology of the total complex and $H^{*}(P ; M)$; cf [13, page 352]. Now, from the definitions of the maps

$$
A^{0, m}(\varphi): A^{0, m}(Q) \rightarrow A^{0, m}(P) \quad \text { and } \quad B^{0, m}\left(\iota_{P}^{Q}\right): A^{0, m}(P) \rightarrow A^{0, m}(Q)
$$

it is easy to check that $H^{n}(A)(\varphi)$ and $H^{n}(B)\left(\iota_{P}^{Q}\right)$ are the usual maps in cohomology of groups; see [4, (D), page 82].

By properties of cohomology for finite groups $\left(H^{n}(A), H^{n}(B)\right): \mathcal{F} \rightarrow \mathbb{Z}_{(p)}$-mod is a cohomological Mackey functor for each $n \geq 0$. Hence so are the functors $\left(F^{n} H^{n+m}(A), F^{n} H^{n+m}(B)\right): \mathcal{F} \rightarrow \mathbb{Z}_{(p)}$-mod induced in the filtration for $n, m \geq 0$. By the arguments above also the pair $\left({ }^{c} E_{\infty}^{n, m}(A),{ }^{c} E_{\infty}^{n, m}(B)\right): \mathcal{F} \rightarrow \mathbb{Z}_{(p)}$-mod is a cohomological Mackey functor for $n, m \geq 0$. Then by Lemma 2.7 we have a short exact sequence of $\mathbb{Z}_{(p)}$-modules

$$
0 \longrightarrow\left(F^{n} H^{n+m}\right)^{\mathcal{F}} \longrightarrow\left(F^{n+1} H^{n+m}\right)^{\mathcal{F}} \longrightarrow{ }^{c} E_{\infty}^{n, m \mathcal{F}} \longrightarrow 0 .
$$

It is immediate that taking invariants and filtering commute and hence we have

$$
0 \longrightarrow F^{n}\left(H^{n+m^{\mathcal{F}}}\right) \longrightarrow F^{n+1}\left(H^{n+m^{\mathcal{F}}}\right) \longrightarrow{ }^{c} E_{\infty}^{n, m \mathcal{F}} \longrightarrow 0
$$

for the filtration of $H^{n+m^{\mathcal{F}}}=H^{n+m}(S)^{\mathcal{F}}$ given by

$$
F^{n}\left(H^{n+m^{\mathcal{F}}}\right)=F^{n}\left(H^{n+m}(S)\right) \cap H^{n+m}(S)^{\mathcal{F}} .
$$

This finishes the proof.

Remark 4.2 We have seen in the proof that for each $2 \leq k \leq \infty$ the pair

$$
\left({ }^{c} E_{k}^{*, *}(A),{ }^{c} E_{k}^{*, *}(B)\right): \mathcal{F} \rightarrow \text { differential bigraded } \mathbb{Z}_{(p)} \text {-modules }
$$

is a cohomological Mackey functor. Moreover, $\left\{\left({ }^{c} E_{k}^{*, *}(A),{ }^{c} E_{k}^{*, *}(B)\right)\right\}_{2 \leq k \leq \infty}$ is a spectral sequence of Mackey functors that converges as a Mackey functor to the usual cohomology of finite groups Mackey functor $\left(H^{*}(A), H^{*}(B)\right): \mathcal{F} \rightarrow \mathbb{Z}_{(p)}-\bmod$.

\section{Comparison}

In this section we compare our spectral sequence and Lyndon-Hochschild-Serre spectral sequence. Let $G$ be a finite group, $K \unlhd G$ and $S \in \operatorname{Syl}_{p}(G)$. Then $T=K \cap S$ is a Sylow $p$-subgroup of $K$. Moreover, $T$ is strongly $\mathcal{F}_{S}(G)$-closed. Fix a $\mathbb{Z}_{(p)}-$ module $M$ with trivial $G$-action. The Lyndon-Hochschild-Serre spectral sequence $E_{*, G}$ of the extension $K \rightarrow G \rightarrow G / K$ is

$$
H^{n}\left(G / K ; H^{m}(K ; M)\right) \Rightarrow H^{n+m}(G ; M)
$$


meanwhile the spectral sequence $E_{*}$ from Theorem 1.1 associated to $T$ is

$$
H^{n}\left(S / T ; H^{m}(T ; M)\right)^{\mathcal{F}_{S}(G)} \Rightarrow H^{n+m}(\mathcal{F} ; M) .
$$

Note that by the classical stable elements theorem, attributed to Tate by Cartan and Eilenberg [6, XII.10.1], $H^{*}(G ; M)=H^{*}(\mathcal{F} ; M)$ and both spectral sequences converge to the same target. Recall that, by construction, $E_{*}$ is a subspectral sequence of the Lyndon-Hochschild-Serre spectral sequence $E_{*, S}$ of $T \rightarrow S \rightarrow S / T$.

Theorem 5.1 The spectral sequences $E_{*, G}$ and $E_{*}$ are isomorphic.

Proof Consider the category $\mathcal{F}_{G}(G)$ with objects the subgroups of $G$ and morphisms given by $\operatorname{Mor}_{\mathcal{F}_{G}(G)}(H, I)=\operatorname{Hom}_{G}(H, I)$. Clearly $\mathcal{F}_{S}(G)$ is a full subcategory of $\mathcal{F}_{G}(G)$. For each subgroup $H \leq G$ we have a short exact sequence

$$
H \cap K \rightarrow H \rightarrow \bar{H}=H / H \cap K .
$$

If $\varphi=c_{g}: H \rightarrow I$ is a morphism in $\mathcal{F}_{G}(G)$ then, as $K$ is normal in $G$, conjugation by $g \in G$ takes $H \cap K \rightarrow H \rightarrow \bar{H}$ to $I \cap K \rightarrow I \rightarrow \bar{I}$. Exactly the same construction of Section 3 gives a cohomological Mackey functor $(A, B): \mathcal{F}_{G}(G) \rightarrow \mathrm{CCh}^{2}\left(\mathbb{Z}_{(p)}\right)$ with values $H \mapsto A^{n, m}(H)=\operatorname{Hom}_{H}\left(\mathcal{B}_{\bar{H}}^{n} \otimes \mathcal{B}_{H}^{m}, M\right)$, where $\mathcal{B}_{H}^{*}$ and $\mathcal{B}_{\bar{H}}^{*}$ are the bar resolutions for $H$ and $\bar{H}$ respectively. Moreover, for $H \leq S$, as $T=K \cap S$, we have $H \cap K=H \cap T$ and this functor over $\mathcal{F}_{G}(G)$ extends the one built in Section 3 over $\mathcal{F}_{S}(G)$.

The inclusion of the short exact sequence $T \rightarrow S \rightarrow S / T$ into $K \rightarrow G \rightarrow G / K$ gives a morphism $\left\{\operatorname{res}_{r}\right\}_{r \geq 2}$ of spectral sequences from $E_{*, G}$ into $E_{*, S}$. The morphism of differential graded algebras $\operatorname{res}_{2}: E_{2, G} \rightarrow E_{2, S}$ coincides with the morphism induced in cohomology by the functor $A$ applied to the inclusion morphism $S \leq G$ of $\mathcal{F}_{G}(G)$, $H^{*, *}(A)\left(\iota_{S}^{G}\right)$. Applying the functor $B$ to the same inclusion $S \leq G$ we get another morphism going in the opposite direction (transfer)

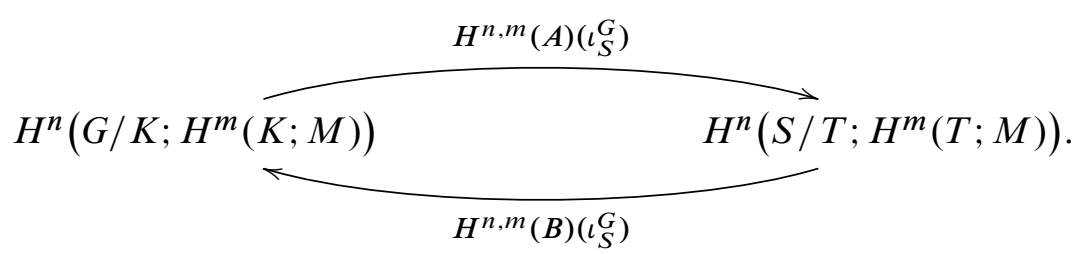

Recall that $E_{2} \leq E_{2, S}$ are exactly the $\mathcal{F}$-stable elements $H^{p}\left(S / T ; H^{q}(T ; M)\right)^{\mathcal{F}_{S}(G)}$. Because conjugation by $g \in G$ induces the identity on $H^{p}\left(G / K ; H^{q}(K ; M)\right)$ it is straightforward that $\operatorname{res}_{2}\left(E_{2, G}\right) \leq E_{2}$. Hence $\left\{\operatorname{res}_{r}\right\}_{r \geq 2}$ is a morphism of spectral sequences $E_{*, G} \rightarrow E_{*}$. If we prove that $\operatorname{res}_{2}\left(E_{2, G}\right)=E_{2}$ then $\operatorname{res}_{2}$ is an 
isomorphism and hence res $r$ is an isomorphism for each $r \geq 2$ and we are done. To see that $\operatorname{res}_{2}\left(E_{2, G}\right)=E_{2}$ we proceed as usual when there is a Mackey functor available; cf [4, Theorem III.10.3]. Let $z \in H^{n}\left(S / T ; H^{m}(T ; M)\right)^{\mathcal{F}_{S}(G)}$ and consider $w=H^{n, m}(B)\left(\iota_{S}^{G}\right)(z) \in H^{n}\left(G / K ; H^{m}(K ; M)\right)$. By the double coset formula 2.2(3) and the cohomological condition 2.2(4) and because $z$ is $\mathcal{F}_{S}(G)$-stable we obtain

$$
\begin{aligned}
H^{n, m}(A)\left(\iota_{S}^{G}\right)(w) & =\sum_{x \in S \backslash G / S} B\left(\iota_{S \cap{ }^{x} S}^{S}\right) A\left(\iota_{S \cap \cap^{x} S}^{x}\right) c_{x^{-1}}^{*}(z) \\
& =\sum_{x \in S \backslash G / S} B\left(\iota_{S \cap{ }^{x} S}^{S}\right) A\left(\iota_{S \cap{ }^{x} S}^{S}\right)(z) \\
& =\sum_{x \in S \backslash G / S}\left|S: S \cap{ }^{x} S\right| z=|G: S| z .
\end{aligned}
$$

As $q=|G: S|$ is a $p^{\prime}$-number it follows that $z=\operatorname{res}\left(\frac{w}{q}\right)$.

Example 5.2 Consider the symmetric group on 6 letters $S_{6}$. It has Sylow 2-subgroup $S=C_{2} \times D_{8}$, where $D_{8}$ is the dihedral group of order 8 . Because $A_{6} \unlhd S_{6}$, the subgroup $T=S \cap A_{6}=D_{8}$ is strongly closed in $\mathcal{F}=\mathcal{F}_{S}\left(S_{6}\right)$. In this example we describe the Lyndon-Hochshild-Serre spectral sequence of $A_{6} \rightarrow S_{6} \rightarrow C_{2}$ interpreted as the spectral sequence $E_{*}^{*, *}$ of Theorem 1.1 applied to $\mathcal{F}$ and $T$. This demonstrates how the new spectral sequence works.

In the fusion system $\mathcal{F}$ there are three $\mathcal{F}$-centric an $\mathcal{F}$-radical subgroups, namely, $S$, $P=C_{2}^{3}$ and $Q=C_{2}^{3}$. The intersections $P \cap T$ and $Q \cap T$ are the two Klein subgroups of $T=D_{8}$. The automorphisms are $\operatorname{Aut}_{\mathcal{F}}(S)=1$ and $\operatorname{Aut}_{\mathcal{F}}(P) \cong \operatorname{Aut}_{\mathcal{F}}(Q) \cong S_{3}$, the symmetric group on 3 letters.

Denote by $E_{*, S}^{*, *}, E_{*, P}^{*, *}$ and $E_{*, Q}^{*, *}$ the Lyndon-Hochschild-Serre spectral sequences of the extensions $T \rightarrow S \rightarrow C_{2}, P \cap T \rightarrow P \rightarrow C_{2}$ and $Q \cap T \rightarrow Q \rightarrow C_{2}$ respectively. All three extensions are direct products and hence all differentials are 0 and the three spectral sequences collapse at the second page. In particular, the ring $H^{*}\left(S ; \mathbb{F}_{2}\right)$ is isomorphic as a ring to $E_{2, S}^{*, *}$ and hence $H^{*}\left(S_{6} ; \mathbb{F}_{2}\right)$ is isomorphic as a ring to $E_{2}^{*, *}$. Moreover, for the invariants we have

$$
E_{2}^{*, *}=E_{2, S}^{*, * \mathcal{F}}=E_{2, S}^{*, *} \cap\left(\operatorname{res}_{P}^{S}\right)^{-1}\left(E_{2, P}^{*, *} S_{3}\right) \cap\left(\operatorname{res}_{Q}^{S}\right)^{-1}\left(E_{2, Q}^{*, *} S_{3}\right),
$$

because it is enough to consider invariants with respect to $\mathcal{F}$-centric and $\mathcal{F}$-radical subgroups by Alperin's fusion theorem. Here,

$$
\operatorname{res}_{P}^{S}: E_{2, S}^{*, *} \rightarrow E_{2, P}^{*, *} \quad \text { and } \quad \operatorname{res}_{Q}^{S}: E_{2, S}^{*, *} \rightarrow E_{2, Q}^{*, *}
$$


are the restriction maps. Denoting by subscripts the degrees we have the following:

$$
\begin{aligned}
& E_{2, S}^{*, *}=H^{*}\left(D_{8} ; \mathbb{F}_{2}\right) \otimes H^{*}\left(C_{2} ; \mathbb{F}_{2}\right)=\mathbb{F}_{2}\left[x_{1}, y_{1}, w_{2}\right] /(x y) \otimes \mathbb{F}_{2}[u], \\
& E_{2, P}^{*, *}=H^{*}\left(C_{2}^{2} ; \mathbb{F}_{2}\right) \otimes H^{*}\left(C_{2} ; \mathbb{F}_{2}\right)=\mathbb{F}_{2}\left[x_{1}, x_{1}^{\prime}\right] \otimes \mathbb{F}_{2}[u], \\
& E_{2, Q}^{*, *}=H^{*}\left(C_{2}^{2} ; \mathbb{F}_{2}\right) \otimes H^{*}\left(C_{2} ; \mathbb{F}_{2}\right)=\mathbb{F}_{2}\left[y_{1}, y_{1}^{\prime}\right] \otimes \mathbb{F}_{2}[u] .
\end{aligned}
$$

Restrictions are given by

$$
\begin{aligned}
& \operatorname{res}_{P}^{S}(x)=x, \quad \operatorname{res}_{P}^{S}(y)=0, \quad \operatorname{res}_{P}^{S}(w)=x x^{\prime}+x^{\prime 2}, \quad \operatorname{res}_{P}^{S}(u)=u, \\
& \operatorname{res}_{Q}^{S}(x)=0, \quad \operatorname{res}_{Q}^{S}(y)=y, \quad \operatorname{res}_{Q}^{S}(w)=y y^{\prime}+y^{\prime 2}, \quad \operatorname{res}_{Q}^{S}(u)=u .
\end{aligned}
$$

Now $S_{3}=\operatorname{Aut}_{S_{6}}(P)$ acts on $P \cap T=C_{2}^{2}$ and on the quotient $C_{2}=P / P \cap T$. The induced action on $H^{*}\left(C_{2}^{2} ; \mathbb{F}_{2}\right)$ is the natural one and on $H^{*}\left(C_{2} ; \mathbb{F}_{2}\right)$ the only possibility is the trivial action. Hence, the invariants are given by

$$
E_{2, P}^{*, *} S_{3}=\mathbb{F}_{2}\left[x_{1}, x_{1}^{\prime}\right]^{S_{3}} \otimes \mathbb{F}_{2}[u]=\mathbb{F}_{2}\left[d_{2}, d_{3}\right] \otimes \mathbb{F}_{2}[u],
$$

where $d_{2}=x^{2}+x^{\prime 2}+x x^{\prime}$ and $d_{3}=\left(x+x^{\prime}\right) x x^{\prime}$ are Dickson's invariants. Analogously, we have that

$$
E_{2, Q}^{*, *} S_{3}=\mathbb{F}_{2}\left[e_{2}, e_{3}\right] \otimes \mathbb{F}_{2}[u]
$$

with $e_{2}=y^{2}+y^{\prime 2}+y y^{\prime}$ and $e_{3}=\left(y+y^{\prime}\right) y y^{\prime}$. It is straightforward that $d_{2}=\operatorname{res}_{P}^{S}\left(x^{2}+w\right), \quad d_{3}=\operatorname{res}_{P}^{S}(x w), \quad e_{2}=\operatorname{res}_{Q}^{S}\left(y^{2}+w\right) \quad$ and $\quad e_{3}=\operatorname{res}_{Q}^{S}(y w)$. From this, it is immediate that $\mathbb{F}_{2}\left[x^{2}+y^{2}+w, x w, y w\right] \otimes \mathbb{F}_{2}[u] \subseteq E_{2}^{*, *}$.

To check the reversed inclusion we first consider stable elements in the polynomial algebras $\mathbb{F}_{2}[x, w]$ and $\mathbb{F}_{2}[y, w]$. As for Long [12, Lemma 1.4.6], the restrictions $\left.\operatorname{res}_{P}^{S}\right|_{\mathbb{F}_{2}[x, w]}$ and $\left.\operatorname{res}_{Q}^{S}\right|_{\mathbb{F}_{2}[y, w]}$ are injective, therefore

$$
\begin{aligned}
& \mathbb{F}_{2}[x, w] \cap\left(\operatorname{res}_{P}^{S}\right)^{-1}\left(E_{2, P}^{*, *} S_{3}\right)=\mathbb{F}_{2}\left[x^{2}+w, x w\right], \\
& \mathbb{F}_{2}[y, w] \cap\left(\operatorname{res}_{Q}^{S}\right)^{-1}\left(E_{2, Q}^{*, *} S_{3}\right)=\mathbb{F}_{2}\left[y^{2}+w, y w\right] .
\end{aligned}
$$

A class $v$ of $H^{n}\left(D_{8} ; \mathbb{F}_{2}\right)$ can be written as follows, where we set $k=\left[\frac{n}{2}\right]$ :

$$
v=\sum_{i=0}^{k} \alpha_{i} w^{i} x^{n-2 i}+\beta_{i} w^{i} y^{n-2 i} .
$$

From the discussion above we have that if $v$ is $\mathcal{F}$-invariant then

$$
v=\epsilon w^{k}+\sum_{2 i+3 j=n} \gamma_{i}\left(x^{2}+w\right)^{i}(x w)^{j}+\delta_{i}\left(y^{2}+w\right)^{i}(y w)^{j},
$$


where $\epsilon=0$ for $n$ odd and $\epsilon=\alpha_{k}+\beta_{k}=\gamma_{k}=\delta_{k}$ if $n$ is even. If $n$ is odd, then the equalities $\left(x^{2}+y^{2}+w\right)(x w)=\left(x^{2}+w\right)(x w)$ and $\left(x^{2}+y^{2}+w\right)(y w)=\left(y^{2}+w\right)(y w)$ give that $v \in \mathbb{F}_{2}\left[x^{2}+y^{2}+w, x w, y w\right]$. If $n$ is even, then the only term left to consider is

$$
\gamma_{k}\left(x^{2}+w\right)^{k}+\delta_{k}\left(y^{2}+w\right)^{k}+\epsilon w^{k}=\gamma_{k}\left(\left(x^{2}+w\right)^{k}+\left(y^{2}+w\right)^{k}+w^{k}\right)
$$

and an easy induction shows that $\left(x^{2}+w\right)^{k}+\left(y^{2}+w\right)^{k}+w^{k}=\left(x^{2}+y^{2}+w\right)^{k}$. So $E_{2}^{*, *}=\mathbb{F}_{2}\left[x^{2}+y^{2}+w, x w, y w\right] \otimes \mathbb{F}_{2}[u]$. The corner of $E_{2}^{*, *}$ is

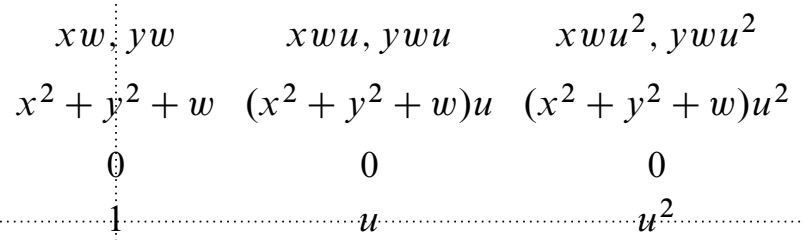

and we deduce that $H^{*}\left(S_{6} ; \mathbb{F}_{2}\right)=\mathbb{F}_{2}\left[u_{1}, a_{2}, b_{3}, c_{3}\right] /(b c)$ with generators

$$
a=x^{2}+y^{2}+w, \quad b=x w \quad \text { and } \quad c=y w .
$$

\section{Stallings' Theorem}

Associated to every first quadrant spectral sequence there is a five-term exact sequence. In the case of the Lyndon-Hochschild-Serre spectral sequence for $K \unlhd G$ and the $G$-module $M$ we obtain the inflation-restriction exact sequence:

$$
\begin{aligned}
0 \rightarrow H^{1}\left(G / K ; M^{K}\right) & \rightarrow H^{1}(G ; M) \\
& \rightarrow H^{1}(K, M)^{G / K} \rightarrow H^{2}\left(G / K ; M^{K}\right) \rightarrow H^{2}(G ; M),
\end{aligned}
$$

where the second arrow from the right is the transgression. Before introducing the five-term exact sequence for the spectral sequence of Theorem 1.1 we introduce some notation. So let $\mathcal{F}$ be a fusion system over the $p$-group $S$ with a strongly closed $\mathcal{F}$-subgroup $T$. Set $[T, \mathcal{F}]=\left\langle[t, \varphi] \mid t \in T, \varphi \in \operatorname{Hom}_{\mathcal{F}}(\langle u\rangle, T)\right\rangle \leq T$, where $[t, \varphi]=$ $t \varphi\left(t^{-1}\right), T^{p}=\left\langle t^{p}, t \in T\right\rangle$, which is characteristic in $T$, and the commutator subgroup $[T, S]=\left\langle t^{-1} s^{-1} t s\right| t \in T$ and $\left.s \in S\right\rangle \unlhd T$. Because the element-wise product $T^{p}[T, S]$ is a normal subgroup of $T$, the element-wise product $T^{p}[S, T] R$ is a subgroup of $T$ for any $R \leq T$. For instance, $T^{p}[T, S][T, \mathcal{F}]=T^{p}[T, \mathcal{F}] \leq T$.

The five-term exact sequence for the spectral sequence of Theorem 1.1 for $\mathcal{F}, T$ and the $\mathbb{Z}_{(p)}$-module $M$ with trivial $S$-action is the following:

$$
\begin{aligned}
0 \rightarrow H^{1}(S / T ; M)^{\mathcal{F}} \rightarrow & H^{1}(\mathcal{F} ; M) \\
& \rightarrow H^{1}(T ; M)^{\mathcal{F}} \rightarrow H^{2}(S / T ; M)^{\mathcal{F}} \rightarrow H^{2}(\mathcal{F} ; M),
\end{aligned}
$$


where the arrow $H^{1}(T ; M)^{\mathcal{F}} \rightarrow H^{2}(S / T ; M)^{\mathcal{F}}$ is the transgression.

For coefficients $M=\mathbb{F}_{p}$ we have

$$
\begin{gathered}
H^{1}\left(\mathcal{F} ; \mathbb{F}_{p}\right)=H^{1}\left(S ; \mathbb{F}_{p}\right)^{\mathcal{F}}=\operatorname{Hom}\left(S / S^{p}[S, \mathcal{F}], \mathbb{F}_{p}\right), \\
H^{1}\left(T ; \mathbb{F}_{p}\right)^{\mathcal{F}}=\operatorname{Hom}\left(T / T^{p}[T, \mathcal{F}], \mathbb{F}_{p}\right) .
\end{gathered}
$$

We also have

$$
\begin{aligned}
& H^{1}(S / T ; M)^{\mathcal{F}}=H^{1}(S / T ; M)^{\mathcal{F} / T}, \\
& H^{2}(S / T ; M)^{\mathcal{F}}=H^{2}(S / T ; M)^{\mathcal{F} / T},
\end{aligned}
$$

by Remark 3.1.

If $\mathcal{F}_{i}$ is a fusion system over the $p$-group $S_{i}$ for $i=1,2$, a homomorphism of groups $\phi: S_{1} \rightarrow S_{2}$ is fusion preserving if for each $\varphi \in \operatorname{Hom}_{\mathcal{F}_{1}}\left(P, S_{1}\right)$ there exists $\hat{\varphi} \in \operatorname{Hom}_{\mathcal{F}_{2}}\left(\phi(P), S_{2}\right)$ such that $\phi \circ \varphi=\hat{\varphi} \circ \phi$. It is easy to see that such a homomorphism induces a map in cohomology $H^{*}\left(\mathcal{F}_{2} ; \mathbb{F}_{p}\right) \rightarrow H^{*}\left(\mathcal{F}_{1} ; \mathbb{F}_{p}\right)$. In fact, by the work of Ragnarsson [15], it induces a map even at the level of stable classifying spaces. Assume, in addition, that $\phi$ induces a map of short exact sequences

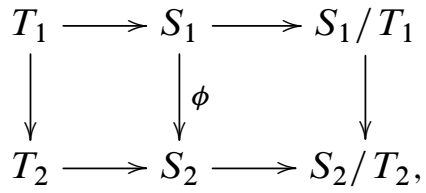

where $T_{i}$ is strongly closed in $S_{i}$ with respect to $\mathcal{F}_{i}$ for $i=1,2$. This is equivalent to assume that $\phi\left(T_{1}\right) \leq T_{2}$. Denote by $E_{i}$ the spectral sequence from Theorem 1.1 applied to the strongly closed subgroup $T_{i}$ for $i=1,2$. Then $\phi$ induces a morphism of spectral sequences $E_{2} \rightarrow E_{1}$ and, in particular, a map of five terms exact sequences.

Theorem 6.1 (Stallings [17]) Let $\mathcal{F}_{i}$ be a fusion system over the $p$-group $S_{i}$ for $i=1,2$ and let $\phi: S_{1} \rightarrow S_{2}$ be a fusion preserving homomorphism. Define

$$
S_{i, 0}=S_{i} \quad \text { and } \quad S_{i, n+1}=S_{i, n}^{p}\left[S_{i, n}, \mathcal{F}_{i}\right] \quad \text { for } i=1,2 \text { and } n \geq 0 .
$$

If the induced map in cohomology $H^{i}\left(\mathcal{F}_{2} ; \mathbb{F}_{p}\right) \rightarrow H^{i}\left(\mathcal{F}_{1} ; \mathbb{F}_{p}\right)$ is isomorphism for $i=1$ and monomorphism for $i=2$ then $S_{1} / S_{1, n} \cong S_{2} / S_{2, n}$ for each $n \geq 1$. In particular, for $n$ big enough we obtain that $S_{1} / \mathcal{O}_{\mathcal{F}_{1}}^{p}\left(S_{1}\right) \cong S_{2} / \mathcal{O}_{\mathcal{F}_{2}}^{p}\left(S_{2}\right)$.

Proof We will prove by induction that $S_{1} / S_{1, n} \cong S_{2} / S_{2, n}$ and that $S_{i, n}$ is strongly $\mathcal{F}_{i}$-closed and contains $O_{\mathcal{F}_{i}}^{p}\left(S_{i}\right)$ for $i=1,2$. For the base case $n=1$, we have that $S_{i, 1}$ contains $O_{\mathcal{F}_{i}}^{p}\left(S_{i}\right)$ and is strongly $\mathcal{F}_{i}$-closed by [8, Corollary A.6] $(i=1,2)$. 
Moreover, by hypothesis, $H^{1}\left(\mathcal{F}_{2} ; \mathbb{F}_{p}\right) \cong H^{1}\left(\mathcal{F}_{1} ; \mathbb{F}_{p}\right)$ and then by Equation (6-3) we get $S_{1} / S_{1,1} \cong S_{2} / S_{2,1}$.

Now let $n \geq 1$. As $\Phi$ is fusion preserving it is easy to see that $\phi\left(S_{1, n}\right) \leq S_{2, n}$. Then we have the following map of short exact sequences:

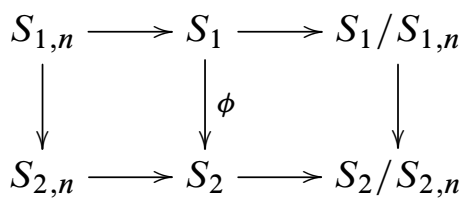

By the induction hypothesis, $S_{1, n}$ and $S_{2, n}$ are strongly closed in $\mathcal{F}_{1}$ and $\mathcal{F}_{2}$ respectively. Then by the discussion before the theorem we have a map of five-term short exact sequences in cohomology with trivial $\mathbb{F}_{p}$-coefficients:

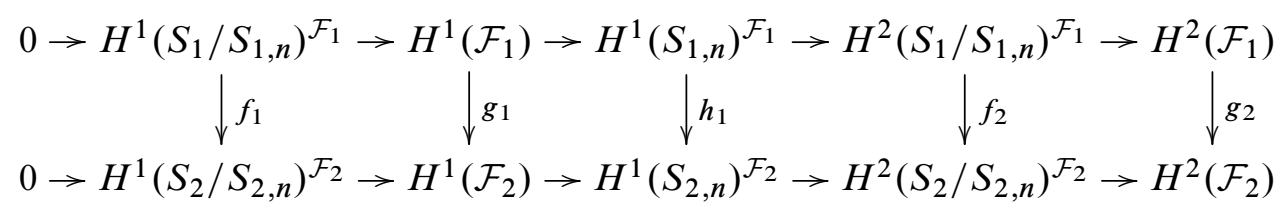

Because $O_{\mathcal{F}_{i}}^{p}\left(S_{i}\right)$ is contained in $S_{i, n}$ the quotient $\mathcal{F} / S_{i, n}$ is a $p$-group, ie,

$$
\mathcal{F} / S_{i, n}=\mathcal{F}_{S / S_{i, n}}\left(S / S_{i, n}\right) \quad \text { for } i=1,2 .
$$

Then, by Equation (6-5), the maps $f_{1}$ and $f_{2}$ are isomorphisms as $S_{1} / S_{1, n} \cong S_{2} / S_{2, n}$. Now, by hypothesis, $g_{1}$ is an isomorphism and $g_{2}$ is a monomorphism. Hence by the five lemma $h_{1}$ is an isomorphism. Then by Equation (6-4) we obtain that $S_{1, n} / S_{1, n+1} \cong S_{2, n} / S_{2, n+1}$ and hence $S_{1} / S_{1, n+1} \cong S_{2} / S_{2, n+1}$.

To finish the induction step, denote by $\mathcal{F}_{i, n}$ the unique $p$-power index fusion subsystem of $\mathcal{F}_{i}$ on $S_{i, n}$ [2, Theorem 4.3]. Then using [8, Lemma A.5] we obtain that $S_{i, n+1}=S_{i, n}^{p}\left[S_{i, n}, S_{i}\right] O_{\mathcal{F}_{i, n}}^{p}\left(S_{i, n}\right)$ and hence, by [8, Corollary A.14],

$$
S_{i, n+1}=S_{i, n}^{p}\left[S_{i, n}, S_{i}\right] O_{\mathcal{F}_{i}}^{p}\left(S_{i}\right) .
$$

Then $S_{i, n+1}$ contains $O_{\mathcal{F}_{i}}^{p}\left(S_{i}\right)$ and by [8, Proposition A.7(1)] $S_{i, n+1}$ is strongly $\mathcal{F}_{i}$-closed for $i=1,2$.

For the second part of the statement recall that for any finite $p$-group $R$ the series $R_{0}=R, R_{n}=R_{n-1}^{p}\left[R_{n-1}, R\right](n \geq 1)$ becomes trivial for $n$ big enough. Then, considering the image of $S_{i, n}$ in $S_{i} / O_{\mathcal{F}}^{p}\left(S_{i}\right)$, it is easy to see that $S_{i, n}=O_{\mathcal{F}}^{p}\left(S_{i}\right)$ for $n$ big enough and $i=1,2$. 
Corollary 6.2 (Evens $[10,7.2 .5]$ ) Let $\mathcal{F}$ be a fusion system over the $p$-group $S$. If the map $H^{2}\left(\mathcal{F} / E_{\mathcal{F}}^{p}(S) ; \mathbb{F}_{p}\right) \rightarrow H^{2}\left(\mathcal{F} ; \mathbb{F}_{p}\right)$ is a monomorphism then $S / O_{\mathcal{F}}^{p}(S)$ is elementary abelian.

Proof Set $\mathcal{F}_{1}=\mathcal{F}$ and $\mathcal{F}_{2}=\mathcal{F} / E_{\mathcal{F}}^{p}(S)$ and consider the fusion preserving quotient map $\mathcal{F}_{1} \rightarrow \mathcal{F}_{2}$. By Equation (6-3) and because $E_{\mathcal{F}}^{p}(S)=\Phi(S) O_{\mathcal{F}}^{p}(S)=S^{p}[S, \mathcal{F}]$, the quotient map induces an isomorphism in degree-1 cohomology. Then Theorem 6.1 gives that $O_{\mathcal{F}}^{p}(S)=E_{\mathcal{F}}^{p}(S)$ and we are done.

Corollary 6.3 (Tate [18]) Let $\mathcal{F}$ be a fusion system over the $p$-group $S$. If the restriction map $H^{1}\left(\mathcal{F} ; \mathbb{F}_{p}\right) \rightarrow H^{1}\left(S ; \mathbb{F}_{p}\right)$ is an isomorphism then $\mathcal{F}=\mathcal{F}_{S}(S)$.

Proof Consider $\mathcal{F}_{1}=\mathcal{F}_{S}(S), \mathcal{F}_{2}=\mathcal{F}$ and the fusion preserving morphism given by inclusion $\mathcal{F}_{1} \subseteq \mathcal{F}_{2}$. Then $H^{1}\left(\mathcal{F} ; \mathbb{F}_{p}\right) \rightarrow H^{1}\left(S ; \mathbb{F}_{p}\right)$ is isomorphism by hypothesis and $H^{2}\left(\mathcal{F} ; \mathbb{F}_{p}\right) \rightarrow H^{2}\left(S ; \mathbb{F}_{p}\right)$ is monomorphism by definition. Then from Theorem 6.1 we obtain $O_{\mathcal{F}}^{p}(S)=1$. Thus there are no $p^{\prime}$-automorphisms in $\mathcal{F}$ and $\mathcal{F}=\mathcal{F}_{S}(S)$.

\section{References}

[1] M Aschbacher, Normal subsystems of fusion systems, Proc. Lond. Math. Soc. 97 (2008) 239-271 MR2434097

[2] C Broto, N Castellana, J Grodal, R Levi, B Oliver, Extensions of p-local finite groups, Trans. Amer. Math. Soc. 359 (2007) 3791-3858 MR2302515

[3] C Broto, R Levi, B Oliver, The homotopy theory of fusion systems, J. Amer. Math. Soc. 16 (2003) 779-856 MR1992826

[4] K S Brown, Cohomology of groups, Grad. Texts in Math. 87, Springer, New York (1982) MR672956

[5] J Cantarero, J Scherer, A Viruel, Nilpotent p-local finite groups (2011) arXiv: $1107.5158 \mathrm{v} 1$

[6] H Cartan, S Eilenberg, Homological algebra, Princeton University Press (1956) MR0077480

[7] D A Craven, Control of fusion and solubility in fusion systems, J. Algebra 323 (2010) 2429-2448 MR2602388

[8] A Díaz, A Glesser, S Park, R Stancu, Tate's and Yoshida's theorems on control of transfer for fusion systems, J. Lond. Math. Soc. 84 (2011) 475-494 MR2835340

[9] A Díaz, A Ruiz, A Viruel, All p-local finite groups of rank two for odd prime $p$, Trans. Amer. Math. Soc. 359 (2007) 1725-1764 MR2272147

[10] L Evens, The cohomology of groups, Clarendon Press, Oxford (1991) MR1144017 
[11] R J Flores, R M Foote, Strongly closed subgroups of finite groups, Adv. Math. 222 (2009) 453-484 MR2538017

[12] J H Long, The cohomology rings of the special affine group of $\mathbb{F}_{p}^{2}$ and of PSL(3,p), PhD thesis, University of Maryland (2008) MR2712167 Available at http:// search.proquest.com//docview/304560975

[13] S Mac Lane, Homology, Springer, Berlin (1995) MR1344215

[14] S Park, Realizing a fusion system by a single finite group, Arch. Math. (Basel) 94 (2010) 405-410 MR2643975

[15] K Ragnarsson, Classifying spectra of saturated fusion systems, Algebr. Geom. Topol. 6 (2006) 195-252 MR2199459

[16] R Solomon, R Stancu, Conjectures on finite and p-local groups, L'Enseignement Mathématique 54 (2008) 171-176

[17] J Stallings, Homology and central series of groups, J. Algebra 2 (1965) 170-181 MR0175956

[18] J Tate, Nilpotent quotient groups, Topology 3 (1964) 109-111 MR0160822

[19] P Webb, A guide to Mackey functors, from: "Handbook of algebra", (M Hazewinkel, editor), North-Holland, Amersterdam (2000) 805-836 MR1759612

Departamento de Álgebra, Geometría y Topología

Campus de Teatinos, Universidad de Málaga, 29071, Málaga, Spain

adiaz@agt.cie.uma.es

http://agt.cie.uma.es/ adiaz

Received: 13 December 2012 Revised: 22 May 2013 